

BNWL-331

\section{COMPUTER CALCULATION OF REACTOR CRAPHITE OXIDATION}

RESEAREH and DEVELOPMENT REPORT



APRIL 1967
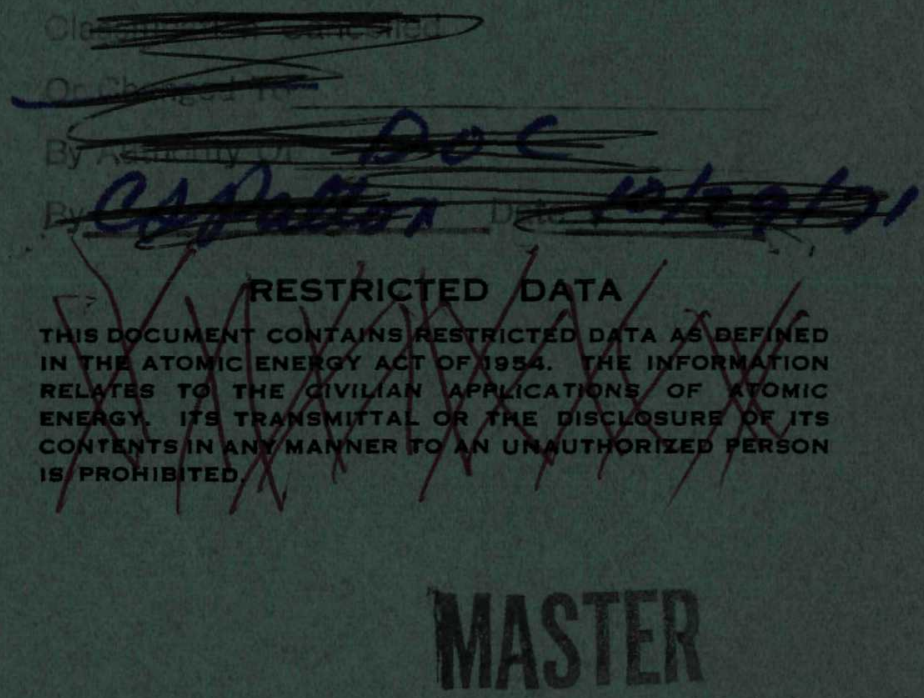

\section{BATTELLE=NORTHWEST}

BATTELLE MEMORIAL. INSTITUTE / PACIFIC NORTHWEST LABORATORY



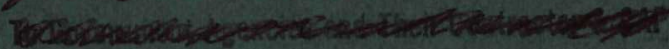

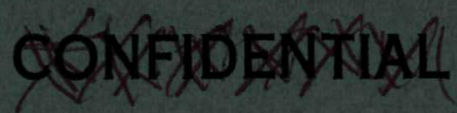

DISTRIBUTION OF. THIS DOCUMENT IS UNLIMITEI 


\section{LEGAL NOTICE}

This report was prepared as an account of Government sponsored work. Neither the United Sitates, nor the Commission, nor any person acting on behalf of the Commission:

A. Makes any warranty or representation, expressed or implied, with respect to the accuracy, completeness, or usefulness of the information contained in this report, or that the use of any information, apparatus, methed, or process disclosed in this report may not infringe privately owned rights; or

B. Assumes any liabilities with respect to the use of, or for damages resulting from the use of any information, apparatus, method, or process disclosed in this report.

As used in the above, "person acting on behalf of the Commission" includes any employee or contractor of the Commission, or employee of such contractor, to the extent that such employee or contractor of the Commission, or employee of such contractor prepares, disseminates, or provides access to, any information pursuant to his employment or contract with the Commission, or his employment with such contractor

\section{PACIFIC NORTHWEST LABORATORY}

RICHLAND, WASHINGTON

operated by

BATTELLE MEMORIAL INSTITUTE

for the

UNITED STATES ATOMIC ENERGY COMMISSION UNDER CONTRACT AT(45-1)-1830 


\section{DISCLAIMER}

This report was prepared as an account of work sponsored by an agency of the United States Government. Neither the United States Government nor any agency Thereof, nor any of their employees, makes any warranty, express or implied, or assumes any legal liability or responsibility for the accuracy, completeness, or usefulness of any information, apparatus, product, or process disclosed, or represents that its use would not infringe privately owned rights. Reference herein to any specific commercial product, process, or service by trade name, trademark, manufacturer, or otherwise does not necessarily constitute or imply its endorsement, recommendation, or favoring by the United States Government or any agency thereof. The views and opinions of authors expressed herein do not necessarily state or reflect those of the United States Government or any agency thereof. 


\section{DISCLAIMER}

Portions of this document may be illegible in electronic image products. Images are produced from the best available original document. 
COMPUTER CALCULATION OF

REACTOR GRAPHITE OXIDATION

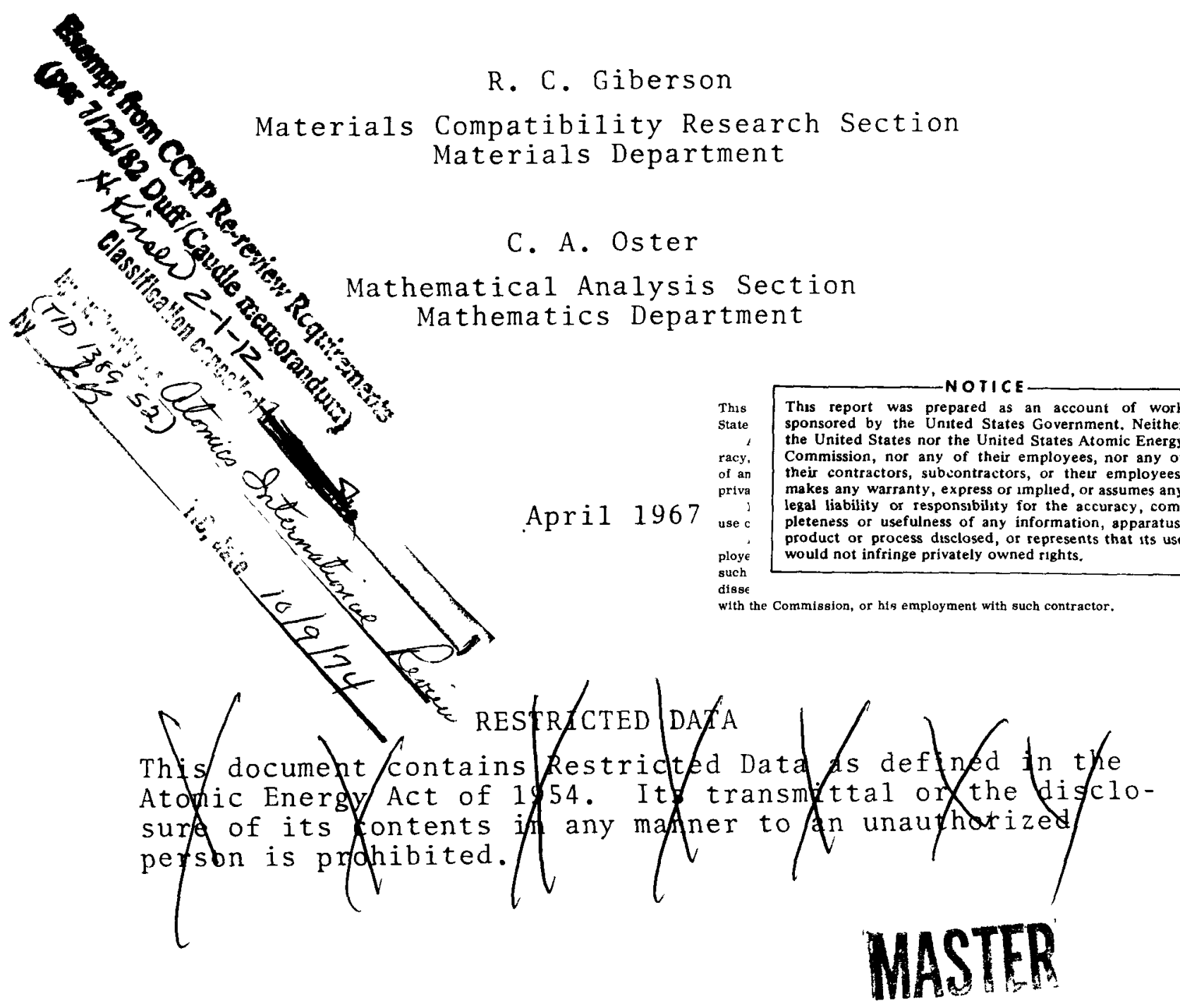

PACIFIC NORTHWEST LABORATORY

RICHLAND, WASHINGTON

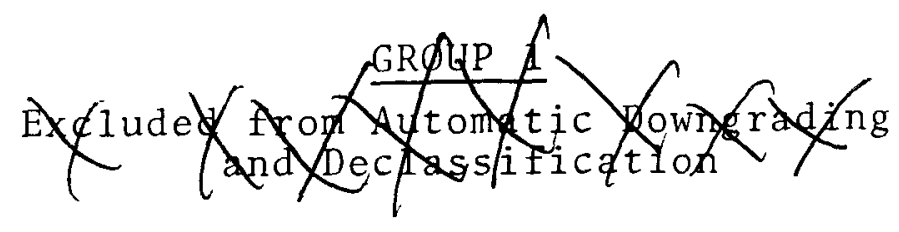

CXIXex 
i i

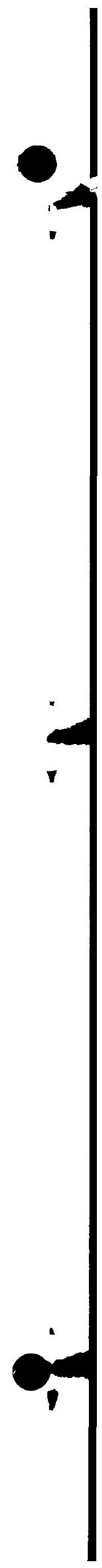






i i i

BNWL - 331

TABLE OF CONTENTS

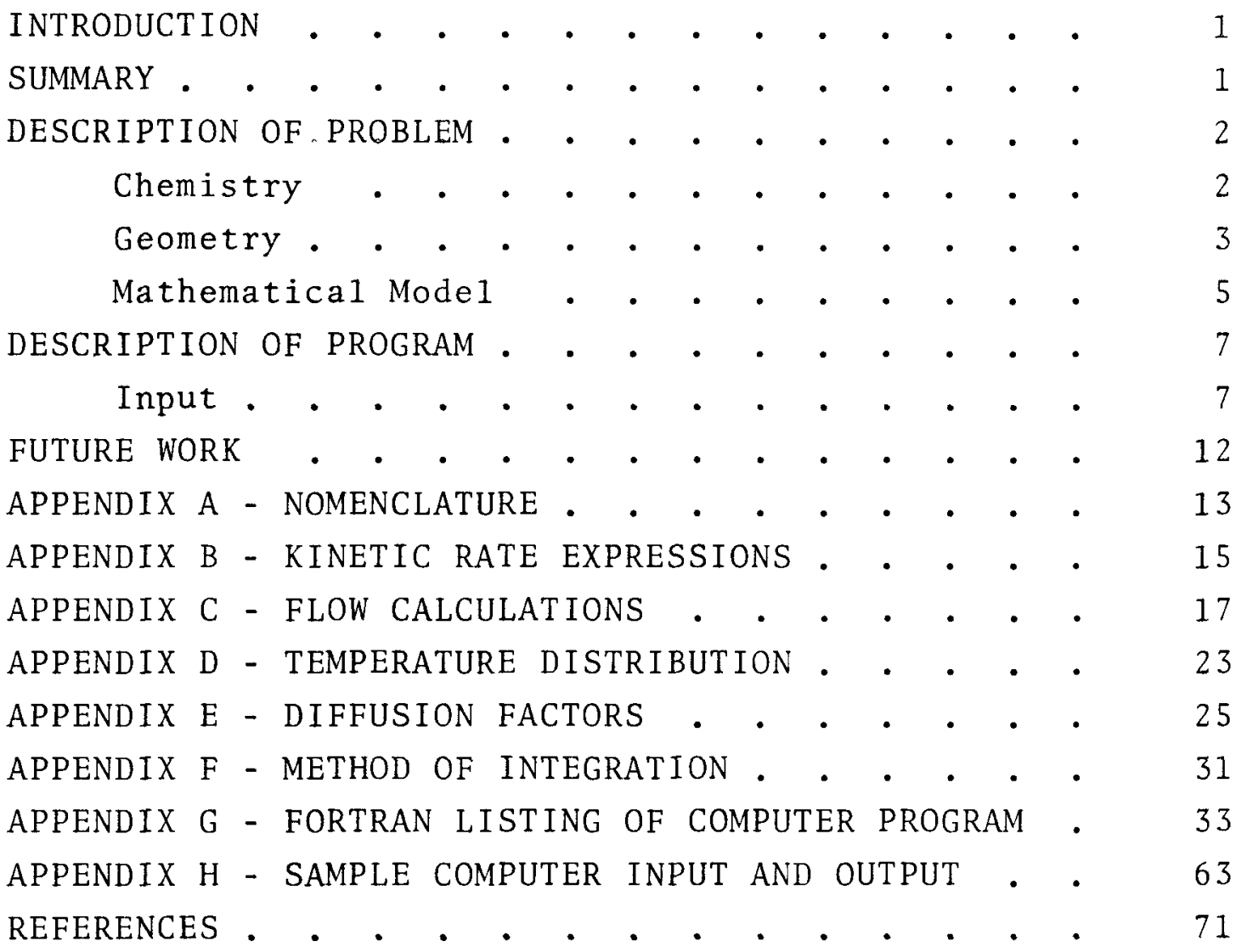

CDOA 


\author{
COMPUTER CALCULATION OF \\ REACTOR GRAPHITE OXIDATION \\ R. C. Giberson and C. A. Oster
}

\title{
INTRODUCTION
}

Graphite oxidation in a graphite-moderated reactor depends on the composition and flow rate of the gas atmosphere, graphite temperature, and geometry of the system. Graphite oxidation, by water vapor or carbon dioxide, in a given system is complicated because of possible inhibition of the oxidation reactions by species such as $\mathrm{H}_{2}$ and $\mathrm{CO}$ and also because gas-phase reactions affect the concentrations of the various gases. Furthermore, diffusion effects in gas-solid reactions must be considered.

A computer program is described in which the rate of graphite oxidation is calculated for a variety of geometrical configurations, and temperature profiles. Chemical reactions involving the gaseous species $\mathrm{H}_{2} \mathrm{O}, \mathrm{CO}_{2}, \mathrm{CO}, \mathrm{H}_{2}$, and $\mathrm{O}_{2}$ are treated. Other chemical reactions involving additional species, such as $\mathrm{CH}_{4}$, can readily be added to the program, which is 1 imited primarily by the lack of knowledge of reaction mechanisms and rate constants.

The program will be illustrated by its application to a low flow, atmospheric pressure oxidation system. However, with relatively minor modifications the program can be adapted to a high flow, high pressure system as in the HTGR.

\section{SUMMARY}

The computer program described calculates graphite oxidation in a variety of geometrical arrangements. Reactions involving the gaseous species $\mathrm{CO}_{2}, \mathrm{CO}, \mathrm{H}_{2}, \mathrm{H}_{2} \mathrm{O}$, and $\mathrm{O}_{2}$ have been included. 
CQDin

\section{DESCRIPTION OF PROBLEM}

The problem, for which the computer solution was developed, can be divided into two parts:

- The chemical problem

- The geometrical problem.

\section{CHEMISTRY}

In a reactor using helium as a gas coolant, a number of impurities may be present. In the present analysis the following gas impurities are considered: $\mathrm{H}_{2} \mathrm{O}, \mathrm{CO}_{2}, \mathrm{CO}, \mathrm{H}_{2}$. and $\mathrm{O}_{2}$. The computer program can easily be modified to consider other possible gases, for example, $\mathrm{CH}_{4}$.

With a complicated gas mixture in contact with graphite, several chemical reactions are possible; and in a nuclear reactor both thermal and radiation processes take place. The reactions currently included in the program are:

Thermal:

$$
\begin{array}{rlll}
\mathrm{C}+\mathrm{H}_{2} \mathrm{O} & \rightarrow & \mathrm{CO}+\mathrm{H}_{2} \\
\mathrm{C}+\mathrm{CO}_{2} & \rightarrow & 2 \mathrm{CO} \\
\mathrm{CO}+\mathrm{H}_{2} \mathrm{O} & \rightarrow & \mathrm{CO}_{2}+\mathrm{H}_{2} \\
\mathrm{CO}_{2}+\mathrm{H}_{2} & \rightarrow & \mathrm{CO}+\mathrm{H}_{2} \mathrm{O} \\
4 \mathrm{C}+3 \mathrm{O}_{2} & \rightarrow & 2 \mathrm{CO}_{2}+2 \mathrm{CO}
\end{array}
$$

Radiation:

$$
\begin{array}{lll}
\mathrm{CO}+\mathrm{H}_{2} \mathrm{O} & \rightarrow & \mathrm{CO}_{2}+\mathrm{H}_{2} \\
\mathrm{CO}_{2}+\mathrm{H}_{2} & \rightarrow & \mathrm{CO}+\mathrm{H}_{2} \mathrm{O}
\end{array}
$$

To calculate the extent of graphite oxidation, mathematical equations are necessary to define the rate of each reaction under the variety of conditions encountered. Appendix $B$ ists the rate expressions for each of the reactions. The nomenclature is given in Appendix A. 
While a number of other reactions are possible, those chosen are considered to be of most significance. Lack of information on rate constants and mechanisms prohibits the addition of other reactions such as the reverse of Reaction 1 and 2 .

\section{GEOMETRY}

The flexibility of the computer system for calculating graphite oxidation is illustrated in Figures 1 and 2 . In the Hanford reactors, oxidation of the graphite is monitored


FIGURE 1. Cross Section View of Graphite Moderator Showing Graphite Burnout Channe? 




FIGURE 2. Longitudinal View of Graphite Containing Channel

with small graphite samples contained in graphite boats, and placed in empty process tube channels of the reactor. Figure 1 shows a cross-sectional view of a typical graphite moderated reactor showing gas passages and a channel containing graphite. A longitudinal view of the channel containing graphite is shown in Figure 2.

The graphite boats are of two types, referred to as "open" or "closed." The open boats (Figure 3) permit direct access of the gas to the small samples. The closed boats (Figure 4) contain both an open and closed section. In the closed section, the gas must diffuse through approximately $1 / 4$ in. of graphite before contacting the samples. Using both types of boats permits measurement of the diffusion effect for the gas-graphite reactions.

The program calculates the oxidation of the boats, samples, and the surrounding moderator along the length of the channel. This calculation requires a knowledge of the concentrations of the various gases and the graphite temperature at any position along the channel. A correction for in-pore gaseous diffusion must be made for all of the gas-graphite reactions. 


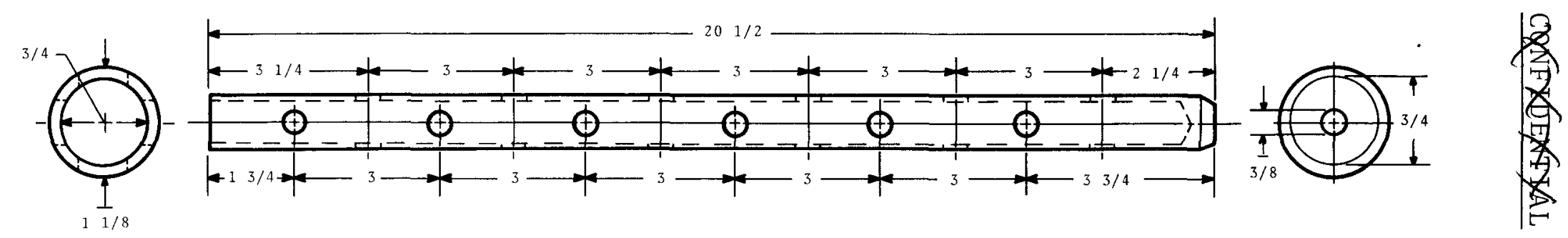

FIGURE 3. Open Graphite Boat (units in inches)
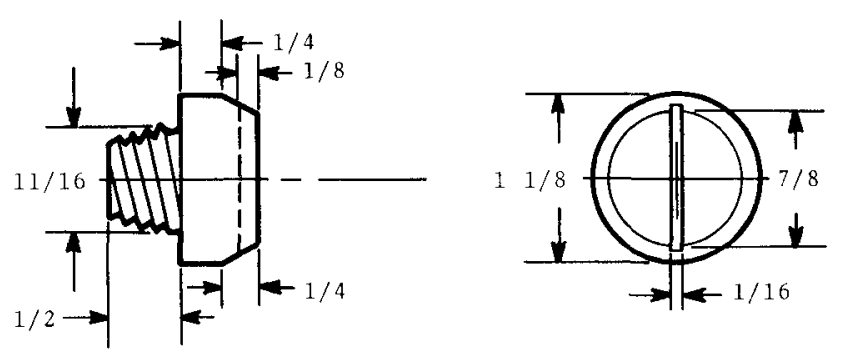
The concentration of gases entering a particular channel depends on the partial pressures of the gases entering the whole reactor system, the total volume flow rate of gas in the system, and the cross-sectional area of the various gas passages. Appendix $C$ shows the detailed calculation of the relative concentrations.

A wide variety of temperature profiles can easily be programmed. In the present case, a simple sine function is used as shown in Appendix D.

The diffusion correction calculations for circular geometry are presented in Appendix E.

MATHEMATICAL MODEL

The information desired from the computer program is the rate of graphite oxidation at any distance along a graphite channe1. This rate of reaction depends on the temperature and gas composition at a given point. The net gas composition is obtained by solving a single differential equation for each gaseous species involved. To illustrate for water vapor:

$$
\frac{\mathrm{d}\left(\mathrm{H}_{2} \mathrm{O}\right)}{\mathrm{dt}}=-\mathrm{R}_{1}-\mathrm{R}_{3}+\mathrm{R}_{4}-\mathrm{R}_{6}+\mathrm{R}_{7}
$$

where $R_{i}$ is the appropriate kinetic expression for the $i^{\text {th }}$ chemical equation 1 isted previously.

In a reactor it is convenient to convert from time dependency to dependence on distance down the channel; thusly,

$$
\frac{\mathrm{d}\left(\mathrm{P}_{\mathrm{H}_{2} \mathrm{O}}\right)}{\mathrm{dx}}=\frac{\mathrm{K} \mathrm{d}\left(\mathrm{H}_{2} \mathrm{O}\right)}{\mathrm{dt}}
$$

where $K$ is the conversion factor.

Finally, in the case of the change of water vapor (or $\mathrm{CO}_{2}$ or $\mathrm{O}_{2}$ ), the diffusion correction must be applied for the gas-solid reaction. This correction also depends on the geometry (Appendix E). 
The differential equation for the change in partial pressure of water vapor versus distance as a result of the watergraphite reaction is:

$$
\frac{\mathrm{d}\left(\mathrm{P}_{\mathrm{H}_{2} \mathrm{O}}\right)}{\mathrm{dx}}=\frac{10^{-5}}{24 \times 3600} \frac{\left(\rho \mathrm{RTR}_{\mathrm{h}}\right)}{\mathrm{M}} \sum\left(\frac{\eta \mathrm{Ag}}{\mathrm{V}}\right)_{i}
$$

where the summation depends on the particular geometrical pattern involved. Appendix A contains an extensive list of nomenclature.

To Equation 8 must be added (or subtracted), the contribution from Reactions $3,4,6$, and 7 . A conversion to the appropriate units of Equation 8 must be made.

These differential equations are solved by a numerical procedure described in Appendix F.

\section{DESCRIPTION OF PROGRAM}

INPUT

The computer program is adaptable to a variety of inputs. Figure 5 shows a flow chart of the program illustrating the various paths of execution. It is possible, for a given geometrical setup, to run various rate constants and/or temperature profiles and, for each set of rate and temperature constants, to run various gas compositions and flow rates. Reactions can be excluded from the program by substituting zeros (or blank cards) for their rate constants. This makes it possible, for example, to separate the effects of thermal and radiation reactions and permits the program to be used for a wide variety of systems. 



FIGURE 5. G. O. P. Computer Flow Logic 
The geometrical factors of the program make it possible, by appropriate choice of constants, to simulate a system as simple as a small cylinder hanging in a quartz tube or an empty channel surrounded by graphite up to a complex reactor system involving burnout boats, chamfer channels, and annular gas passages.

A description of the input deck follows:

$\begin{array}{cl}\frac{\text { Card No. }}{1} & \frac{\text { Forma }}{(12 \mathrm{~A} 6)} \\ 2 & \text { (I5) }\end{array}$

\section{Contents of Card}

Run identification.

Print frequency. One line of output will be printed each time the number of integration steps is a multiple of this number, e.g., if print frequency is 1, every step will be printed; if it is 3 , then steps $3,6,9$, would be printed. In addition the conditions at each XM (See card $7+N$ ) will be printed.

(3 (6XA6), Reactor identification, channel 4A6) identification, oxidation test number, date charged, date discharged.

No. of chamfer channels, No. of annular channels, No. of burnout channels.

(6E12.8) CE - Edge measurement of a chamfer flow channel.

DE - Diameter of empty burnout channel. DPT - Outside diameter of process tube. ODB - Outside diameter of boat. DOBI - Inside diameter of boat. DS - Outside diameter of sample. (A11 of these measurements are in in inches). 


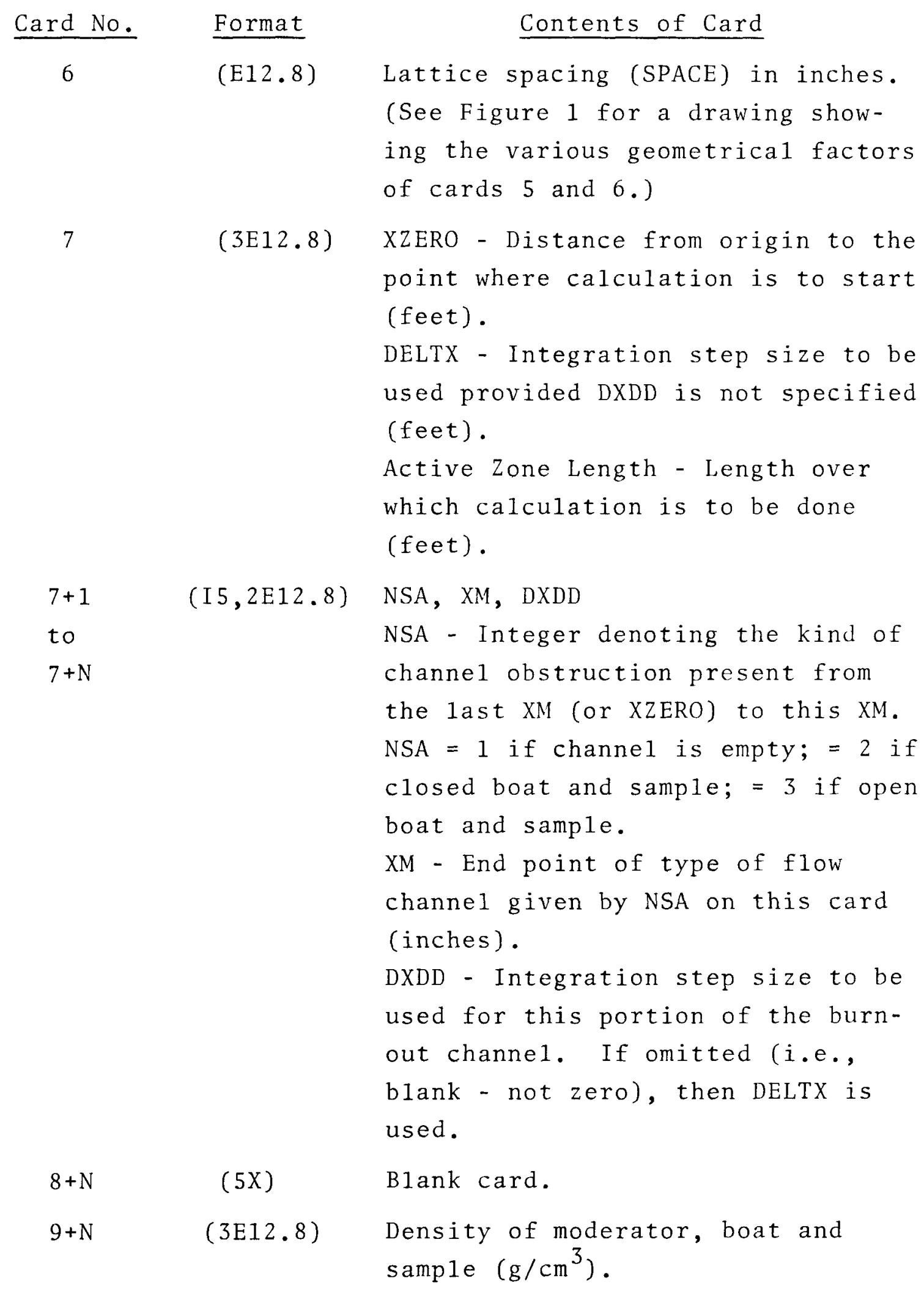


This completes the first section of the input deck. The next section is all under an E12.8 format (maximum of 6E12.8 per card) except for card No. 21. Cards $10+N$ through $19+N$ are rate constant cards. Card $20+\mathrm{N}$ is the temperature card and card 21 (3I5) is a switching card.

\begin{tabular}{|c|c|c|}
\hline Card No. & Reaction & Contents of Card \\
\hline $10+N$ & $\mathrm{CO}+\mathrm{H}_{2} \mathrm{O}$ & $\begin{array}{l}\text { First activation energy, first } \\
\text { frequency factor, second activation } \\
\text { energy, second frequency factor, } \\
\text { inhibition factor. }\end{array}$ \\
\hline $11+N$ & $\mathrm{CO}_{2}+\mathrm{H}_{2}$ & Same as card $10+\mathrm{N}$ \\
\hline $12+\mathrm{N}$ & $\mathrm{C}+\mathrm{H}_{2} \mathrm{O}$ & $\begin{array}{l}\text { First, second and third activation } \\
\text { energies. }\end{array}$ \\
\hline $13+N$ & $\mathrm{C}+\mathrm{CO}_{2}$ & Same as card $12+\mathrm{N}$ \\
\hline $14+N$ & $\mathrm{C}+\mathrm{H}_{2} \mathrm{O}$ & $\begin{array}{l}\text { First, second and third frequency } \\
\text { factors, diffusion factor, tempera- } \\
\text { ture coefficient for diffusion } \\
\text { factor. }\end{array}$ \\
\hline $15+N$ & $\mathrm{C}+\mathrm{CO}_{2}$ & Same as card $14+N$. \\
\hline $16+N$ & $\begin{array}{l}\mathrm{CO}+\mathrm{H}_{2} \mathrm{O} \\
(\mathrm{Gamma})\end{array}$ & $\begin{array}{l}\text { G-value, activation energy, dose } \\
\text { rate, efficiency factors one and } \\
\text { two. }\end{array}$ \\
\hline $17+N$ & $\begin{array}{l}\mathrm{CO}_{2}-\mathrm{H}_{2} \\
\text { (Gamma) }\end{array}$ & $\begin{array}{l}\text { G-value, activation energy, effi- } \\
\text { ciency factors one and two. }\end{array}$ \\
\hline $18+N$ & $\mathrm{C}+\mathrm{O}_{2}$ & $\begin{array}{l}\text { Frequency factor, activation } \\
\text { energy, order of reaction, diffu- } \\
\text { sion factor, temperature coefficient } \\
\text { of diffusion. }\end{array}$ \\
\hline $19+\mathrm{N}$ & $\begin{array}{l}\mathrm{C}+\mathrm{O}_{2} \\
(\mathrm{Gamma})\end{array}$ & $\begin{array}{l}\text { Frequency factor, activation energy, } \\
\text { order of reaction. }\end{array}$ \\
\hline
\end{tabular}




\begin{tabular}{|c|c|c|}
\hline Card No. & Reaction & Contents of Card \\
\hline $20+N$ & $\begin{array}{l}\text { Temperature } \\
\text { Card }\end{array}$ & $\begin{array}{l}\text { Inlet temperature (i.e., room } \\
\text { temperature), initial graphite } \\
\text { temperature, maximum graphite, } \\
\text { temperature, extrapolation } \\
\text { distance (feet). Temperatures } \\
\text { are given in degrees Centigrade. }\end{array}$ \\
\hline $21+N$ & $\begin{array}{l}\text { Switching } \\
\text { Card }\end{array}$ & $\begin{array}{l}\text { IPLOT - If negative or zero, no } \\
\text { plot is made. If positive, a } \\
\text { plot is made on the printer. } \\
\text { IEXIT - If negative or zero, } \\
\text { return is to beginning of input } \\
\text { deck. If positive, return is } \\
\text { to second section of input deck } \\
\text { making it possible to run multi- } \\
\text { ple rate constant variations and/or } \\
\text { temperature variations for the } \\
\text { same geometrical setup. } \\
\text { IETA - If zero or negative, no } \\
\text { effect. If positive, the values } \\
\text { of eta are printed out. }\end{array}$ \\
\hline
\end{tabular}

Third Section - Input concentrations and flow rates. $22+\mathrm{N}(6 \mathrm{E} 12.8) \quad \mathrm{PH}_{2} \mathrm{O}, \mathrm{PCO}, \mathrm{PCO}_{2}, \mathrm{PH}_{2}, \mathrm{~V}, \mathrm{PO}_{2}$. $22+N+M \quad$ Last card must be blank or all zeros. As many partial pressure cards as desired may be used. The pressures are given in millimeters mercury. The $V$ is volume flow in cubic feet per minute total for the system.

A detail listing of the program is found in Appendix $G$ and a sample input-output using hypothetical conditions is shown in Appendix $\mathrm{H}$. 


\section{FUTURE WORK}

The program will be quantitatively tested by a controlled temperature and gas composition experiment conducted in a furnace $8 \mathrm{in}$. in diameter by $9 \mathrm{ft}$. long.

The current program is limited by a knowledge of rate constants and mechanism for other possible reactions. As this type of information becomes available the program can be expanded. 
APPENDIX A

NOMENCLATURE 


\section{APPENDIX A}

NOMENCLATURE

General

$\mathrm{Ag}$

$\mathrm{C}$

D

$\mathrm{I}_{\mathrm{n}}$

$\mathrm{K}_{\mathrm{n}}$

${ }^{\mathrm{k}} \mathrm{v}$

$\ell$

$\mathrm{L}$

$\mathrm{M}$

$\mathrm{N}$

$\mathrm{P}$

$\mathrm{p}$

$\mathrm{R}$

r

$\mathrm{T}$

$\mathrm{V}$

$\mathrm{X}$

Z

Other Notation

$\triangle$

$\delta$

ก

$\rho$

$\nabla$ cross-sectional area of graphite

gas concentration

flow channel diameter

modified Bessel Function of the 1st kind, order $\mathrm{n}$

modified Bessel Function of the 2nd kind, order $\mathrm{n}$

rate constant

flow channel length

oxidation depth

molecular weight of carbon

number of flow channels (or boats)

atmosphere pressure

partial pressure

reaction rate if subscripted, universal gas constant otherwise

radius measurement

temperature

volume flow rate

distance measurement

diffusion coefficient

used to indicate an increment

reactor extrapolation distance

diffusion factor

graphite density

The Laplacian differential operator 
Subscripts

Subscripts are used to identify individual quantities within a given class. The following subscripts and their meaning are used.

$\begin{array}{ll}\text { A } & \text { annular flow channel } \\ \text { B } & \text { burnout flow channel } \\ \text { b } & \text { boat } \\ \text { C } & \text { chamfer flow channel } \\ \text { C } & \text { closed boat } \\ \text { E } & \text { reactor lattice edge measurement } \\ \text { e } & \text { empty flow channel (void of boats) } \\ \text { eff } & \text { "effective" relationship or quantity } \\ \text { ex } & \text { exterior quantity } \\ \text { h } & \text { homogeneous } \\ \text { i } & \text { interior quantity } \\ \mathrm{m} & \text { moderator } \\ \text { o } & \text { open boat } \\ \mathrm{p} & \text { process tube } \\ \mathrm{S} & \text { sample } \\ \mathrm{T} & \text { total or composite quantity for system }\end{array}$

Superscripts

In relation to the closed and open boats there are two gas concentrations. In this case the superscripts $i$ and ex are used to indicate interior and exterior quantities respectively. 
APPENDIX B

KINETIC RATE EXPRESSIONS

Water-Graphite $^{(1)} \quad \mathrm{H}_{2} \mathrm{O}(\mathrm{g})+\mathrm{C}_{(\mathrm{s})} \rightarrow \mathrm{CO}_{(\mathrm{g})}+\mathrm{H}_{2}(\mathrm{~g})$

$$
\mathrm{R}_{\mathrm{h}}=\frac{\mathrm{k}_{1} \mathrm{P}_{\mathrm{H}_{2} \mathrm{O}}}{1+\mathrm{k}_{2} \mathrm{P}_{\mathrm{H}_{2}} 1 / 2+\mathrm{k}_{3} \mathrm{P}_{\mathrm{H}_{2} \mathrm{O}}}
$$

Carbon Dioxide-Graphite ${ }^{(2)} \mathrm{C}_{(\mathrm{s})}+\mathrm{CO}_{2}(\mathrm{~g}) \rightarrow{ }^{2 \mathrm{CO}}(\mathrm{g})$

$$
\mathrm{R}_{\mathrm{h}}=\frac{\mathrm{k}_{1} \mathrm{P}_{\mathrm{CO}}}{1+\mathrm{k}_{2} \mathrm{P}_{\mathrm{CO}}{ }^{+} \mathrm{k}_{3} \mathrm{P}_{\mathrm{CO}}}
$$

Water-gas shift reaction (forward) $(3)$

$$
\mathrm{R}=\frac{{ }^{\mathrm{CO}}(\mathrm{g})+\mathrm{H}_{2} \mathrm{O}(\mathrm{g}) \rightarrow \mathrm{CO}_{2}(\mathrm{~g})+\mathrm{H}_{2}(\mathrm{~g})}{\left(\mathrm{T}+\mathrm{aP}_{\mathrm{H}_{2} \mathrm{O}}\right)^{1 / 2} \mathrm{P}_{2} \mathrm{O}}
$$

Water-gas shift reaction (reverse) (4)

$$
\begin{gathered}
\mathrm{CO}_{2}(\mathrm{~g})+\mathrm{H}_{2}(\mathrm{~g}) \rightarrow \mathrm{CO}_{(\mathrm{g})}+\mathrm{H}_{2} \mathrm{O}(\mathrm{g}) \\
\mathrm{R}=\mathrm{k}_{1} \mathrm{P}_{\mathrm{CO}}{ }_{2} \mathrm{P}_{\mathrm{H}_{2}} 1 / 2+\mathrm{k}_{2} \mathrm{P}_{\mathrm{CO}_{2}} \mathrm{P}_{\mathrm{H}_{2}} 1 / 3
\end{gathered}
$$

Oxygen-graphite reaction (2)

$$
4 \mathrm{C}(\mathrm{s})+30_{2}(\mathrm{~g}) \rightarrow 2 \mathrm{CO}_{2}(\mathrm{~g})+2 \mathrm{CO}(\mathrm{g})
$$

$$
\mathrm{R}_{\mathrm{h}}=\mathrm{k}_{\mathrm{P}_{\mathrm{O}_{2}}} \mathrm{n}
$$

The k's in the above reactions are exponential functions of temperature:

$$
k=k_{0} e^{-E / R T}
$$


Radiation Reactions - The yields of radiation-induced reactions are commonly expressed as $G$ values (molecules reacted or formed per $100 \mathrm{eV}$ of energy ahsorbed). Reactions 6 and 7 have been studied by G. L. Tingey. $(5,6)$ The rate expression is:

$$
\mathrm{R}=\mathrm{Ge} \mathrm{e}^{-\mathrm{E} / \mathrm{RT}} \mathrm{gF}
$$

where $g$ is the radiation dose rate in Roentgens per hour and $F$ is a constant containing appropriate conversion factors as well as gas pressure terms to correct for energy absorbed in the various gases and $E$ is the activation energy for the radiation-induced process. 
APPENDIX C

FLOW CALCULATIONS 


\section{APPENDIX C \\ FLOW CALCULATIONS}

To calculate graphite weight loss in a given location, it is necessary to determine what portion of the total gas flow, $V$, flows in the channel of interest.

Assumptions used in deriving the relationships in the program are as follows:

I. The mass flow is conservative, i.e., for a given time increment the same quantity of gas flows into the system as flows out.

II. The mass flow rate is proportional to the fourth power of the flow channel diameter.

III. The mass flow rate is directly proportional to the product of the pressure drop $\Delta \mathrm{P}$ over the flow channel and the fourth power of the flow channel diameter divided by the flow channel length.

IV. The effective radius of a flow channel is taken to be the so-called hydraulic radius or twice the ratio of the area of flow to the wetted perimeter of the flow channel.

V. The mass flow rate between two surfaces is assumed to be split into two channels by a fictitious surface between the two actual surfaces in such a manner that half the total mass flow rate is in each channel.

Consider the boat loaded channel as shown in Figure 2, where all open boats are contiguous as are the closed boats.

Let $D_{0}$ eff denote the effective diameter of the open boatsample combination. ( $D_{0}$ eff is defined in Equation C-8 below.) 
From Assumption I, we have

$$
\mathrm{V}_{\mathrm{B}}=\mathrm{V}_{\mathrm{c}}=\mathrm{V}_{\mathrm{O}}=\mathrm{V}_{\mathrm{e}}
$$

and from Assumption II,

$$
V_{B}=\frac{K D_{B}^{4} \Delta P_{B}}{\ell_{B}}=\frac{K D_{c}^{4} \Delta P_{c}}{\ell_{c}}=\frac{K D_{o}^{4} \text { eff } \Delta_{0}}{\ell_{o}}=\frac{K D_{e}^{4} \Delta P_{e}}{\ell_{e}}
$$

Now

$$
\begin{aligned}
& \Delta \mathrm{P}_{B}=\Delta \mathrm{P}_{C}+\Delta \mathrm{P}_{0}+\Delta \mathrm{P}_{\mathrm{e}} \text {, which by using Equation } \mathrm{C}-1 \text { leads to } \\
& \Delta \mathrm{P}_{\mathrm{B}}=\frac{\mathrm{V}_{\mathrm{B}}}{\mathrm{K}}\left\{\frac{\ell_{\mathrm{C}}}{\mathrm{D}_{\mathrm{C}}^{4}}+\frac{\ell_{\mathrm{o}}}{\mathrm{D}_{\mathrm{o}}^{4} \text { eff }}+\frac{\ell_{\mathrm{e}}}{\mathrm{D}_{\mathrm{e}}^{4}}\right\} .
\end{aligned}
$$

Thus

$$
\frac{V_{B}}{D_{B}^{4}}=\frac{K \Delta P_{B}}{\ell_{B}}=\frac{V_{B}}{\ell_{B}} \quad\left\{\frac{\ell_{c}}{D_{c}^{4}}+\frac{\ell_{o}}{D_{o}^{4} \text { eff }}+\frac{\ell_{e}}{D_{e}^{4}}\right\} \text {. }
$$

Or on solving for the effective diameter of a boat loaded channel we have

$$
\mathrm{D}_{\mathrm{B} \text { eff }}^{4}=\ell_{\mathrm{B}}\left\{\frac{\ell_{\mathrm{c}}}{\mathrm{D}_{\mathrm{c}}^{4}}+\frac{\ell_{\mathrm{o}}}{\mathrm{D}_{\mathrm{o} \text { eff }}^{4}}+\frac{\ell_{\mathrm{e}}}{\mathrm{D}_{\mathrm{e}}^{4}}\right\}^{-1}
$$

Now from Assumption II it follows that

$$
\frac{\mathrm{V}_{\mathrm{C}}}{\mathrm{D}_{\mathrm{C}}^{4}}=\frac{\mathrm{V}_{\mathrm{A}}}{\mathrm{D}_{\mathrm{A}}^{4}}=\frac{\mathrm{V}_{\mathrm{B}}}{\mathrm{D}_{\mathrm{B}}^{4} \text { eff }}
$$

which leads to

$$
V_{B}=\frac{V_{C}}{D_{C}^{4}}=D_{B}^{4} \text { eff }
$$


and

$$
\mathrm{V}_{\mathrm{A}}=\frac{\mathrm{V}_{\mathrm{C}}}{\mathrm{D}_{\mathrm{C}}^{4}} \mathrm{D}_{\mathrm{A}}^{4}
$$

Assumption I gives the relationship

$$
\mathrm{V}_{\mathrm{T}}=\mathrm{N}_{\mathrm{C}} \mathrm{V}_{\mathrm{C}}+\mathrm{N}_{\mathrm{A}} \mathrm{V}_{\mathrm{A}}+\mathrm{N}_{\mathrm{B}} \mathrm{V}_{\mathrm{B}}
$$

which from Equations $\mathrm{C}-2$ and $\mathrm{C}-3$ yields

$$
\mathrm{V}_{\mathrm{C}}=\mathrm{V}_{\mathrm{T}}\left[\mathrm{N}_{\mathrm{C}}+\frac{1}{\mathrm{D}_{\mathrm{C}}^{4}}\left(\mathrm{~N}_{\mathrm{A}} \mathrm{D}_{\mathrm{A}}^{4}+\mathrm{N}_{\mathrm{B}} \mathrm{D}_{\mathrm{B} \text { eff }}^{4}\right)^{-1}\right. \text {. }
$$

In the case of the annular channe1, Assumption IV implies that

$$
D_{A}=2\left(r_{e}-r_{p e x}\right)
$$

The chamfer flow channels are assumed to have cross sections which are right isosceles triangles. Here the flow area is $E_{C}^{2} / 2$ and the wetted perimeter is $(2+\sqrt{2}) E_{C}$. Hence, by Assumption IV,

$$
{ }^{D} \mathrm{C}=\frac{2}{2+\sqrt{2}^{E}} \mathrm{C} \text {. }
$$

In treatment of flow within the burnout channel, the channel is assumed to have the form shown in Figure 2. Using Assumption IV, we have

$$
\begin{aligned}
& D_{b i \text { eff }}=2\left(r_{b i}-r_{s}\right) \\
& D_{b \text { ex eff }}=2\left(r_{e}-r_{b \text { ex }}\right) .
\end{aligned}
$$

If the boat is closed, then there is only one flow channel, in which case

$$
D_{c \text { eff }}=D_{b} \text { ex eff }
$$


Consider the open boat and sample case. Here, by Assumption $\mathrm{V}$,

$$
\mathrm{V}_{\mathrm{o}}=\mathrm{V}_{\mathrm{o} \text { ex }}+\mathrm{V}_{\mathrm{o} i}=1 / 2 \mathrm{~V}_{\mathrm{B}}
$$

and, from Assumption II,

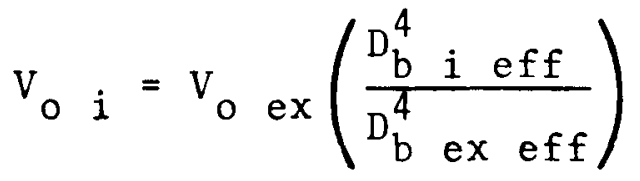

which with Equation C-7 leads to.

$$
V_{o \text { ex }}=V_{B}\left(\frac{D_{b}^{4} \text { ex eff }}{D_{b}^{4} \text { ex eff }+D_{b}^{4} \text { i eff }}\right) \text {. }
$$

Equation C-6 may also be written as

$$
\frac{V_{o \text { ex }}}{D_{b}^{4} \text { ex eff }}=\frac{V_{B}}{D_{b}^{4} \text { ex eff }+D_{b}^{4} \text { eff }} .
$$

Using Assumption II again and Equation C-5, we have

$$
\frac{V_{B}}{D_{b}^{4} \text { ex eff }}=\frac{V_{B}}{D_{o}^{4} \text { eff }}
$$

or by solving for $D_{0}$ eff

$$
D_{o \text { eff }}=\left(D_{b}^{4} \text { ex eff }+D_{b}^{4} \text { i eff }\right)^{1 / 4} \text {. }
$$

Calculation of burnout rates for the moderator, boat, and sample requires Assumption $V$ which gives

Case 1. Empty channe1

$$
\mathrm{V}_{\mathrm{me}}=\mathrm{V}_{\mathrm{B}}
$$

Case 2. Closed boat

$$
\begin{aligned}
& \mathrm{v}_{\mathrm{mc}}=1 / 2 \mathrm{~V}_{\mathrm{B}} \\
& \mathrm{v}_{\mathrm{bc}}=1 / 2 \mathrm{~V}_{\mathrm{B}}
\end{aligned}
$$


Case 3. Open boat

$$
\begin{aligned}
& \mathrm{V}_{\text {mo }}=\mathrm{V}_{\text {o ex }} \\
& \mathrm{V}_{\text {so }}=\mathrm{V}_{\text {oi }} \\
& \mathrm{V}_{\text {bo }}=\mathrm{V}_{\text {mo }}+\mathrm{V}_{\text {so }} .
\end{aligned}
$$




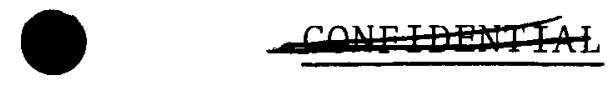

BNWL - 331

APPENDIX D

TEMPERATURE DISTRIBUTION 


\section{APPENDIX D \\ TEMPERATURE DISTRIBUTION}

A temperature distribution of the following form has been programmed.

$T=a+b \sin [(X+\delta) s]+273.16$

where:

$$
\begin{aligned}
\mathbf{s} & =\frac{\Pi}{2\left(\ell^{\prime}+\delta\right)} \\
\mathrm{b} & =\frac{\text { TMAX }-\mathrm{TIN}}{1-\mathrm{SIN}(\delta \mathrm{S})} \\
\mathrm{a} & =\text { TMAX }-\mathrm{b} \\
\ell^{\prime} & =\text { active zone length } \\
\delta & =\text { extrapolation } 1 \text { ength } \\
\mathrm{TIN} & =\text { inlet temperature }\left({ }^{\circ} \mathrm{C}\right) \\
\text { TMAX } & =\text { maximum graphite temperature }\left({ }^{\circ} \mathrm{C}\right) .
\end{aligned}
$$


APPENDIX E

DIFFUSION FACTORS 


\section{APPENDIX E}

\section{DIFFUSION FACTORS}

In gas-solid reactions, it is necessary to account for the diffusion of the gas into and away from the solid. The effect of diffusion is taken into account through the so-called depth of oxidation $L$ :

$$
L=\left(\frac{Z}{k_{v}}\right)^{1 / 2}
$$

$Z$ is the effective diffusion coefficient:

$Z=m D_{12}$

where $D_{12}$ is the actual gaseous diffusion coefficient and $m$ is a variable that depends on the type of material. Graphite values of $\mathrm{m}$ from 0.004 to 0.01 have been determined.

The variable $k_{v}$ is the specific volume rate constant (i.e., volume of carbon removed per volume of graphite present per unit time) for the reaction. It is defined for the water graphite reaction:

$$
\mathrm{k}_{\mathrm{v}}=\overline{\mathrm{K}} \frac{\mathrm{R}_{\mathrm{h}} \mathrm{T} \rho}{\mathrm{P}_{\mathrm{H}_{2} \mathrm{O}}} \sec ^{-1}
$$

The constant $\bar{K}$ is for unit conversion and is given by

$$
\overline{\mathrm{K}}=6.01019 \times 10^{-7}\left(\frac{\mathrm{mm} \mathrm{Hg}}{\mathrm{K}}\right)\left(\frac{\mathrm{cm}^{3}}{\mathrm{~g}}\right)\left(\frac{\mathrm{KOD}}{\frac{0}{0}}\right)\left(\frac{1}{\mathrm{sec}}\right) \text {. }
$$

The solution of the diffusion equation for circular geometry is shown below. The assumptions involved in the solution are:

I. A gas concentration, $C$, in contact with a material diffuses into that material according to the diffusion equation, i.e.,

$$
\nabla^{2} \mathrm{C}=\frac{1}{\mathrm{~L}^{2}} \mathrm{C} .
$$


Two sets of coefficients must be determined for the closed boat case: one set for the exterior and one set for the interior. Let

$$
\begin{aligned}
& \Omega=\left(\frac{L^{e x}}{L^{i}}\right) I_{1}\left(r_{e x}^{i} / L^{i}\right) K_{0}\left(r_{i}^{e x} / L^{e x}\right)+I_{O}\left(r_{e x}^{i} / L\right) K_{i}\left(r_{i}^{e x} / L^{e x}\right) \\
& x=I_{0}\left(r_{e x}^{i} / L^{i}\right) I_{1}\left(r_{i}^{e x} / L^{e x}\right)-\left(\frac{L^{e x}}{L^{i}}\right) I_{I}\left(r_{e x}^{i} / L^{i}\right) I_{0}\left(r_{i}^{e x} / L^{e x}\right) \\
& \psi=\Omega I_{0}\left(r_{e x}^{e x} / L^{e x}\right)+x K_{o}\left(r_{e x}^{e x} / L^{e x}\right)
\end{aligned}
$$

and

$$
\zeta=\frac{I_{0}\left(r_{e x}^{i} / L^{i}\right)}{\left(r_{i}^{e x} / L^{e x}\right) \psi}
$$

then,

$$
\begin{aligned}
A^{e x} & =\frac{C_{\text {ex }}^{e x}}{\psi} \Omega \\
B^{e x} & =\frac{C_{e x}^{e x}}{\psi} x \\
A^{i} & =\zeta C_{e x}^{e x} / I_{0}\left(r_{e x}^{i} / L^{i}\right) \\
B^{i} & =0
\end{aligned}
$$

The effective reaction rate $R_{\text {eff }}$ is the volume integral of $q=k_{v} c$. For a cylinder of unit length, we have

$$
R_{\text {eff }}=k_{v} \int_{0}^{1} \int_{0}^{2 \pi} \int_{r_{i}}^{r} \text { ex } r d r d \theta d x
$$

Under Assumption II, this expression reduces to

$$
R_{e f f}=2 \pi k_{v} \int_{r_{i}}^{r} \operatorname{ex}(r) r d r
$$


Now Equation E-1 is used with Equation E-4 and E-6 to obtain

Notice that

$$
\begin{aligned}
R_{\text {eff }}= & \frac{2 \pi Z}{L}\left\{r_{e x}\left[A I_{1}\left(r_{e x} / L\right)-B K_{1}\left(r_{e x} / L\right)\right]\right. \\
& \left.-r_{i}\left[A I_{1}\left(r^{i} / L\right)-B K_{1}\left(r_{i} / L\right)\right]\right\} .
\end{aligned}
$$

$$
R_{\text {eff }}=2 Z\left[\left.\begin{array}{ll}
r & \frac{d C}{d r}
\end{array}\right|_{r=r_{\text {ex }}}-\left.\left(r \frac{d C}{d r}\right)\right|_{r=r_{i}}\right]
$$

The homogeneous reaction rate, $R_{h}$, is defined in a similar manner except that $C$ is further assumed to be independent of $r$;

$$
\begin{aligned}
R_{h} & =k_{v} C_{e x} \int_{0}^{1} \int_{0}^{2 \pi} \int_{r}^{r} e^{i} r d r d \theta d x \\
& =\pi k_{v} C_{e x}\left(r_{e x}^{2}-r_{i}^{2}\right) .
\end{aligned}
$$

The diffusion factor, $n$, is defined to be the ratio of $R_{\text {eff }}$ to $R_{h}$;

$$
\eta=\frac{R_{\text {eff }}}{R_{h}} \text {. }
$$

In the case of the closed boat, Equation E-7 gives $n$ under the condition of $\mathrm{C}_{\mathrm{ex}}=\mathrm{C}_{\mathrm{i}}$; however, Equation $\mathrm{E}-5$ is simply the ratio $\mathrm{C}_{1} / \mathrm{C}_{\mathrm{ex}}$. Therefore, we use the following relationship to obtain

$$
n_{c s}=\zeta \frac{R_{\text {eff }}}{R_{h}}
$$

Hence, there are from one to four diffusion factors to calculate depending on the geometry of the flow channel. 
APPENDIX F

METHOD OF INTEGRATION 


\section{APPENDIX F}

\section{METHOD OF INTEGRATION}

Vector notation will be used to describe the numerical integration procedure. Let $y^{i}$ denote the solution of the $i^{\text {th }}$ differential equation $\frac{d y^{i}}{d x}=f^{i}\left(x, y^{i}\right)$. Now let

$$
\vec{y}=\left(\begin{array}{c}
y_{2}^{i} \\
y^{2} \\
\cdot \\
y^{s}
\end{array}\right) \text { and } \vec{f}(x, \vec{y})=\left(\begin{array}{cc}
f^{1} & (x, \vec{y}) \\
f^{2} & (x, \vec{y}) \\
\cdot \\
\cdot \\
f^{s}(x, \vec{y})
\end{array}\right)
$$

Other vectors are similarly defined. The initial conditions are denoted by the subscript $o$. If $h$ is a constant, let $x_{i}$ $=x_{i-1}+h$ and let $\vec{y}_{i}$ denote the solution vector $\vec{y}$ at the

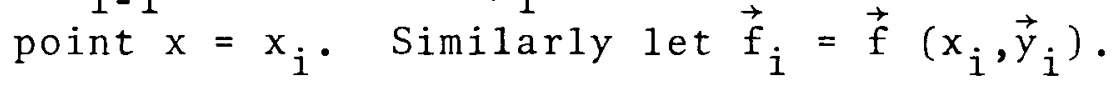

The numerical procedure used is a combination of Runge-Kutta and predictor-corrector methods. The RungeKutta method is used to obtain starting data required by the predictor-corrector procedure. The Runge-Kutta scheme utilized may be described as follows: (7)

$$
\begin{aligned}
& \overrightarrow{\mathrm{k}}_{1}=\overrightarrow{\mathrm{h}} \mathrm{f}_{0} \\
& \overrightarrow{\mathrm{k}}_{2}=\mathrm{h} \overrightarrow{\mathrm{f}}\left(\mathrm{x}_{0}+\frac{\mathrm{h}}{2}, \vec{y}_{0}+\frac{\overrightarrow{\mathrm{k}}}{2}\right) \\
& \overrightarrow{\mathrm{k}}_{3}=\mathrm{h} \overrightarrow{\mathrm{f}}\left(\mathrm{x}_{0}+\frac{\mathrm{h}}{2}, \vec{y}_{0}+\frac{\overrightarrow{\mathrm{k}}_{2}}{2}\right) \\
& \overrightarrow{\mathrm{k}}_{4}=\mathrm{h} \overrightarrow{\mathrm{f}}\left(\mathrm{x}_{0}+\mathrm{h}, \vec{y}_{0}+\overrightarrow{\mathrm{k}}_{3}\right) \\
& \vec{y}_{1}=\vec{y}_{0}+\frac{1}{6}\left(\overrightarrow{\mathrm{k}}_{1}+2 \overrightarrow{\mathrm{k}}_{2}+2 \overrightarrow{\mathrm{k}}_{3}+\overrightarrow{\mathrm{k}}_{4}\right)
\end{aligned}
$$

This algorithm is repeated three times giving $\vec{y}_{1}, \vec{f}_{1}, \vec{y}_{2}, \vec{f}_{2}$, $\vec{y}_{3}$ and $\vec{f}_{3}$. After the points $\vec{y}_{0}, \vec{y}_{1}, \vec{y}_{2}$, and $\vec{y}_{3}$ have been 
determined, subsequent points are obtained by the following predictor-corrector method.

Milnes predictor formula is

$$
\vec{y}_{4}=\vec{y}_{0}+\frac{4 h}{3}\left(2 \vec{f}_{1}-\vec{f}_{2}+2 \vec{f}_{3}\right)
$$

and Hamming's corrector formula

$$
\vec{y}_{4}=1 / 8\left[9 \vec{y}_{3}-\vec{y}_{1}+3 h\left(\vec{f}_{4}+2 \vec{f}_{3}-\vec{f}_{2}\right)\right]
$$

Equation (F-1) is used repeatedly until successive iterates agree to 7 significant figures. The solution at $x_{5}$ is obtained by adjusting the subscripts and applying the predictor equation again, followed by repeated applications of the corrector equation. If it is necessary to change the value of $h$, the Runge-Kutta procedure is repeated again at that point. 
APPENDIX G

FORTRAN LISTING OF COIMPUTER PROGRAM 
COOO GRAPHITE OXIDATION PROGRAM

DIMENSION PO2(5), DRVO2(5)

DIMENSION AI $(5,3), B 1(5), C 1(5), D 1(5,3), X(5)$, DRVHZO(5), DRVCO(5),

IDRVCO2 (5), PH2O(5), PCO(5),PCO2 (5), PH2 (5),

2DENT (12), TYPE(2), NSAI (50), XMI (50), DXDDI $(50), T Y P(2,50)$

COMMON ZUH2O, ABH2O, NSAMPL, ZOCO2, ABCO2, YI

COMMON $\triangle$, EXT, VKODC, VKODB, VKODS, AVS

COMVION AVC, AVB, TY, UGC, R , RE

CONMON RO, RB, RS

$\begin{array}{lll}\text { - UGC } & \text {, R } & \text {, RE } 10, H 2 O K 20, H 20 \times 30\end{array}$

COMMON H2OX1, $\mathrm{H}_{2} \mathrm{O} \times 2, \mathrm{H}_{2} \mathrm{O} \mathrm{X}_{3}, \mathrm{CO} 2 \mathrm{KLO}, \mathrm{CO} 2 \mathrm{~K} 2 \mathrm{O}, \mathrm{CO} 2 \mathrm{~K} 3 \mathrm{O}$

COMMON CO2XI, $C O 2 \times 2, \operatorname{CO} 2 \times 3$, TA, TB, AKRK1

COMGON AKRK2, AKRK3, AKRK4, ALRK1, ALRK2, ALRK3

COMMON ALKK4, ANRK1, AMRK2, AMRK3, AMRK4, KRK

COMMON DRVH2O, DRVCO, DRVCO2, PH2O, PH2, PCO

COMMON PCO2, AI, BI, $\mathrm{B}$, $\mathrm{Cl}, \mathrm{DI}, \mathrm{BIEXI}$

COMMON BIEX2, CIEXI, CIEX2, BIFFCI, BIFFC2, GIFFCI

COMVON CIFFC2, OIIFA, CIIFA, HALFVO, VCDO, RBT

COMVIMON $V B$, $X$, Y, TYI, TKV, TK

COMMON ZCO2, ZH2O, BICUN, CICONI, DENMW, DENS

COMMON DENB, R1, R3, EHZO1, EH2O2, EH2O3

COMMION ECO21, ECO22, ECO23, NSAI, XMI, DXDDI

COMMON HZOK1, H2OK2, H2OK3, COZK1, CO2K2, CO2K3

COMMON DENT, TYPE, TYP, RBSQ, RDSQ, ZCTK

COMINON AM, AB , AS, RATIOL, EHZOT, ECOZT

COMMON PCKOD, TIN, TMAX, BORC, BORB, BORS

COMNON H2OL, CO2L, HZOKV, CO2KV, ANRKI, ANRK2

COMMON ANKK3, ANRK4, DKVU2, PO2, O2KI, O2K2

COMWON APW, BPW, O2KV, O2L, EO2I, EO23

COMMON EL22, EO2T, ZO2, ABO2, ZOO2, O2XI

COMMON O2X2, O2K10, OZK20, GE, DELE, XKRAD

COMMON ACON, GVALUE, REXP, VFACT, TEFAC, WWA

COMMON ECAREA, CGAREA, OBAREA, TIK, RGE, RDELE

0000000

0000010

0000020

0000030

0000040

0000050

0000060

0000070

0000080

0000090

0000100

0000110

0000120

0000130

0000140

0000150

0000160

0000170

0000180

0000190

0000200

0000210

0000220

0000230

0000240

0000250

0000260

0000270

0000280

0000290

0000300

0000310

COMMON RACON, RGVAL, CICON2, WI

0000320

COMPON / ANA/CFICM, IQ, JQ, KQ, RQUCH(450), RWOOS(450), RQOCS(450), DQOCH(0000330 $1450), D 200 S(453)$, DQOCS(450)

0000340

COMMON /JAN/ EH2OCH(450), EH2OSA $(450)$, ECO2CH(450), ECO2SA (450), EO2CHO000350 $X(450), E C 2 S A(450), H 2 O L P(450), C O 2 L P(450), 02 L P R(450)$, PRTYP $1(450)$, PRTYOO000360 XP2(45U), TYIPR $(450)$, NIP(450) 0000370

COMMUN / JUKLS/ JUPHZ, JUUXY, JUCU, JUHYU, JUSHZU, JUSCU2, JOCO2

0000360

1 FORMAT $(1 \mathrm{HI} / / / / / / / / / / / 22 \times 12 \mathrm{A6}, / / / 18 \times 11 \mathrm{HREACTOR}$ KEYIOXA6,10XI6HDATEO000390 1 CHARGEU 2A6,/18X14HCHANNEL NUMBER7XA6,10X16HDATE DISCHARGED 2 A0000400 $26, / 18 \times 21$ HBLRNOUT TEST NUMBER A6)

2 FORMAT $(3(6 \times 46), 4 A 6)$

3 FORMAT $(1 H+117 X, 14,11)$

4 FORMAT(IH 2 A6,F9.2, 5 FIU.4,F10.0, IP3E12.3)

5 FORMAT (12A6)

6 FORMAT $(14$ I5)

7 FORMAT $(15,2 E 12.8)$

9 FORMAT (6EI2.8) INOUTF $8.3 / / 1$

202 FORMATIIIUHU CHANNEL

$1 E S$

2LE

INCHES

H20

DISTANCE

CHAMFERF8.3,9H ANNULARF8.3,9H BURO000490

0000410

0000420

0000430

0000440

0000450

0000460

0000470

3HTEMP. - . , 5X, 7HCHANAEL, 6X, 4HBOAT, 7X, GHSANPLE

0000500

203 FORMAT( 34 HUNO. OF BOATS ETC. EXCEEDS LIMITS )

204 FORMAT (IHUF8.4, IX2A6,FY.4, IX2AG,F7.4, IX2A6,F9.4, IX $\angle A 6, F 9.4,1 \times 2 A 6$,

IF9.4/(IUX2A6,F9.4, I X2A6,F9.4,IX2A6,F9.4, IX2A6,F9.4,1X2A6,F9.41) $B U R N O U T$ R A T E S/6OH PROFIOOOO520
CO CO2, $8 \times, 2 H O 2,4 X, 80000530$

0000540 0000550 0000560 2 U5 FORMATI 4 SHUNU. OF GHAVIFER FLOW CMANNLLS............... $5.16 \times 39 H L D 0000500$ 
IGE OF GHAMFLR FLOW CHANNEL..........F7.4/45H NU. OF ANNULAK FLUW 0000590 2LHANNELS............... 15,18X39HDIAMETLR LF EMPTY FLOW CHANNEL..000006UO 3......F7.4/45H NO. UF GURNOUT FLOW CHANIVLS............... 2,18X30000610 49HOUTSILE DIAMETER UF PROCESD TLEL.......F7.4/68X9YHOUTSIDE DIAMETO000620 5LR UF BOHT.............F7.4/43H REACTOK LATTILL SPACINU.......0000630


73 A ACTIVE ZONE LENUTH (FELT)....................18X39HUIANETER 00000650 $8 F$ SAMPLE......................... 7.4 ) 0000660

206 FORIATIIHI25X27HRATE CALCULATION PARAMETERS//26X8HCO + H2O17X8HCO20000670 $1+$ H238X16HCARSON DENSITIES/24XIHIIUXIH214XIHIIOXIH2/18H ACTIVATI00000680 2N ENERGYIP2E11.3,4X2E11.3,3UX 3 HMODERATOROPF9.4/18H FREQUENGY FACT00000690 $3 \mathrm{~F}$ IP $2 E 11 .=, 4 \times 2 E 11.3,30 \times 4 \mathrm{HBOAT} 5 \mathrm{X}$ UPF9.4/18H INHIBITION FACTUK $7 \times F 9.0000700$ $44,17 \times F 9.4,36 \times 9 H S A M P L E \quad F 9.4 / 1)$

207 FORMAT $(2$ IHUTEMPERATURES GASF6.1,3XISHGKAPHITE INLETF8.2, $9 H \quad 0000720$ IMAXIMUMFO.2, I2X2LHEXTRAPOLATION DISTANGEF7.31 10000730

$2 \cup 8$ FORMAT $127 \mathrm{H}$ INITIAL CONDITIONS FLOW $=F 7.2,8 \mathrm{H}$ PH2O $=F 8.3,7 \mathrm{H} \quad$ PFO $=0000740$ $1 F 8.3,8 \mathrm{H} \quad \mathrm{PCO} 2==8.3,7 \mathrm{H} \quad \mathrm{PH} 2=\mathrm{F} 6.3,7 \mathrm{H} \quad \mathrm{PO} 2=\mathrm{F} 8.3 / 11 \quad 0000750$

200 FORMAT $(1 H 1,22 \times 12 A G /)$ 0000760


I7HFACTORS//18H ACTIVATION ENERGYIPE11.3,2E15.3,9X2HZOE10.3/18H FREO000780 2QUENCY FACTOR EI1.3, $\angle 215.3,9 \times 3 H A T 2 O P F 9.4 / 11$ 0000790

211 OFORMAT (IHU, 47HOATA FUR THE CO + H2O REACTIUN IN A GAMNA FIELO/11HOOOUOZUO $1,3 H G E=, 1 P E I U .3,5 X, 5 \mathrm{HDELE}=, \mathrm{H} 10.3,5 \times, 6 \mathrm{H} \times K R A D=, E 10.3,5 \mathrm{HACON}=, \mathrm{H} 10.3,250000810$ $2 X, 6 H I P L O T=, I 3,2 X, 6 H I E X I T=, I 3,2 X, 5 H I E T A=, 13 / 11$ 0000820

212 UFORMATIIHU,29X,35HJATA FOR OXYGEN - GRAPHITE REACTION/12X,36HFREQU0000830 IENCY FACTOR ACTIVATION ENEROY,IUX, SHORUER, 17X, I7HDIFFUSION FACTCO000840 2RS/ $6 \mathrm{H}$ THERMAL, 5X,7HU2KIU =,IPEIU.3,ICH U2EI $=, E 10.3,10 \mathrm{H}$ APW0000850 $3=, E I U .3, I U H \quad Z U U 2=, E I O .3,10 \mathrm{H} \quad$ ABO2=,EIO.3/20H RADIATION 00000860 42K2U =, EIU.3, IUH O2E2=,E10.3,1UH BPW =,E10.3/1 0000870

213 UFOKIAATILHU, 47HDATA FUR THE CO2 + H2 REACTIUN IN A GAMMA FIELD, $47 \times, 0000800$ 113 HPRINT FREQ. =, I $/ / 1 H O, 4 H R G L=I P E I U .3,5 X, 6 H R D E L E=, E 10.3,5 X, 7 H R A C 000008$ O $2 N=, E I-.3,37 X, 11 H O T E P$ SIZE $=, E 10.4 / 1$


1,15HL(DEPTH) VALUES//32H CHANNEL DIJTANCE WATER, $8 X, 14 H C A R 0000920$ 2BON DIOXIDE, $8 X, 6 H O X Y C E N, 13 X, 26 H W A T E R$ CARBCN OXYGEN,8X,4HTEMOOOO930 3P/7IH PRO.ILE INCHES CHAN. SAMP. CHAN. SAMP. CHOOOO940 4AN. SAMP.,22X,7HDIOXIDE, $18 X, 2 \mathrm{HC}, / / 1 \mathrm{IH}, 2 \mathrm{AG}, \mathrm{F} 9.4,2 \mathrm{~F} 7.4,4 \mathrm{X}, 2 \mathrm{~F} 7.4,4 \times 0000950$ $5,2 F 7.4,8 \times, 1 P 3 E 10.3,5 \times$, OPF 5.011 0000960

216 LFOKMATIIHU,IIUHIF THE CONSTANTS BELOW HAVE A VALUE OF I THE INDICAOOOOY7O ITED REACTION WAS SHUT DOWN BECAUSE OF LOW GAS CONCENTRATION/ $18 H$ J00000980 $2 \mathrm{PHZ}=$, IL, 48त HYDROGEN INHIDITION OF WATER GRAPHITE REACTION/8H J00000990 $3 U X Y=, I b, 26 H$ OXYGEN-GRAPHITE RLACTION/8H JUCO =, I5,18H CO + H200001000 4 THERMAL/8H JOHYD $=, 15,18 \mathrm{H}$ CO2 + H2 THERMAL/8H JOSH2O=, I5,20H COOOOIOIO $5+$ H2O RADIATION/8H JOSCOL=, I5, $2 \mathrm{CH} \mathrm{CO2+H2} \mathrm{RADIATICN/8H} \mathrm{JOCO2=,0001020}$ 6I5,45H CD + H2O RADIATION HYDROGEN WENT O OR MINUS 1 OCOIO3O CFICM IS THE CONVEKSION FACTOR FOR INCHES TO CENTIMETERS 0001040 SEE NAT. DUREAU OF STAND. MANLBOOK OF MATH. FUNCTIONS, APP. MATH. OOO1050 SERIES NO. 55, JUNE 64, PP 7-8

ATJ IS A CONVERSION CONSTANT (JOULES/(ATMOS*C:1\%*3))

CAJ IS A CONVERSIUIV CONSTANT (JULLES/CALORIE)

RGC IS THE UNIVERSAL GAS CUNSTANT (JOULES/ (DEG KriULE))

CATMWT IS THE GRAN ATOMIC WT UF CAKBUN (GM/MOLE) ATMOS IS ATMUSPHEKIC PKESSUKE AT JEA LEVEL (MM OF HG) ZCTK IS O DEG K EXPRESSED IN DEG C ABSOLUTE ZERO. TIMEPC 15 THE BASIC TIME UNIT RATIO (O/O*SEC/KOD) MAXLIN IS THE NUMBER OF LINES PER PAGE UF OUTPUT DATA PI/3.14159265/,FORCPI/1.27323454/,CFICM/2.540005/, IATJ/.IC13250/,CAJ/4.1840/,RGC/8.3143/,CATMWT/12.01/,ATMOS/760./, $2 Z C T K / 273.16 /$, TIMEPC/8.64E+9/,MAXLIN/45/ 0001060 0001070 0001080 $00010 y 0$ 0001100 0001110 0001120 0001130 0001140 0001150 0001160 0001170 


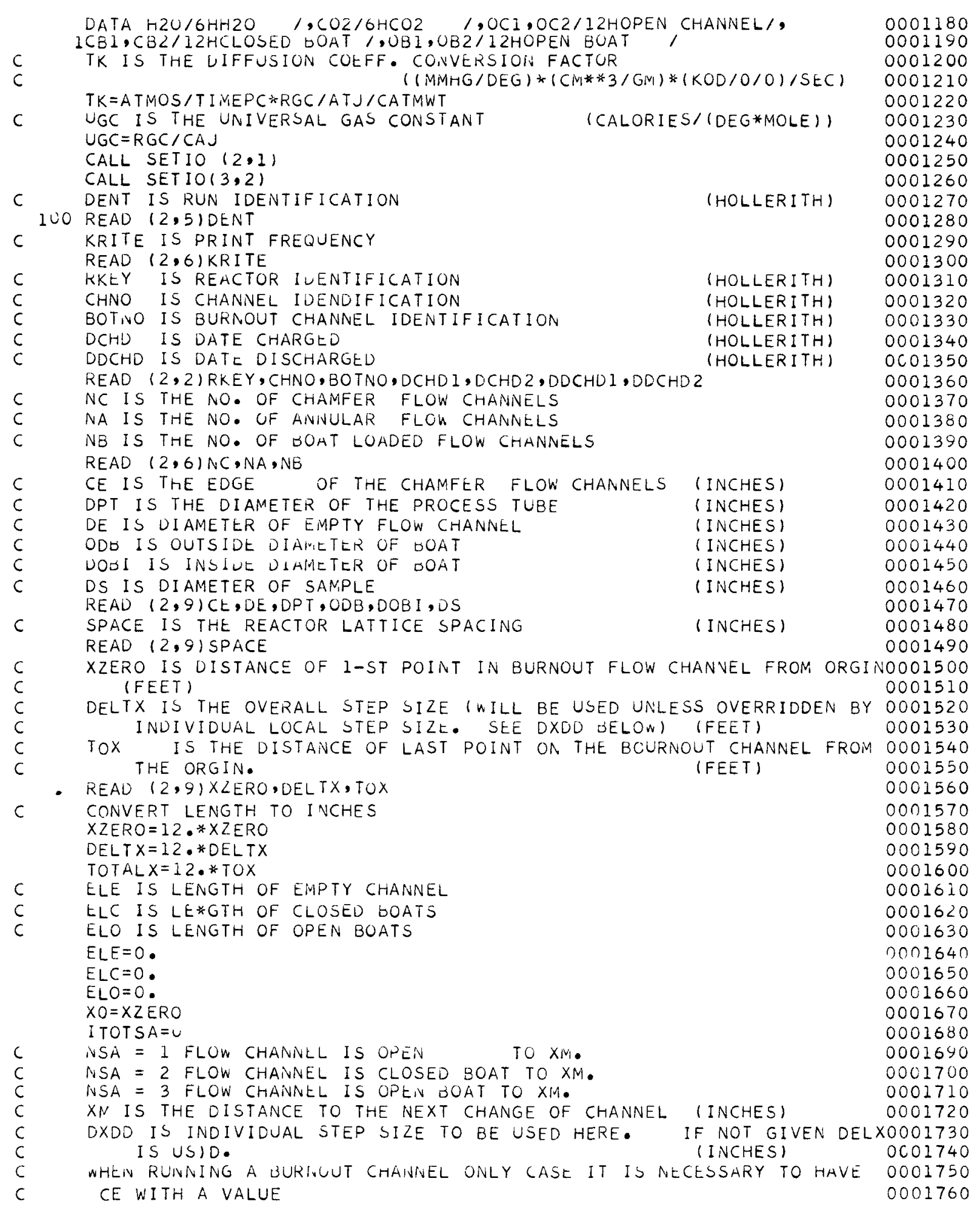


IF (NC) $51,51,30$

0001770

$51 C E=1.0$

3U REAU $(2,7) N S A, X M, D X D D$

IF (NSA) $31,31,130$

130 I TOT SA $=$ I TOTSA+1

IF (I TOTSA-5U) $230,230,330$

0001780

0001790

0001800

0001810

0001820

$230 X M=A M I N 1(X M$, TOTALX)

$O I F L=X M-X O$

$X O=X M$

$X M I(I T O T S A)=X M$

DXDDI (ITOTSA) $=$ DXDD

NSA $=$ MINO (MAXO (NSA, 1),3)

NSAI ( I TOTSA) $=N S A$

GO TO $(73 \cup, 830,930)$, NSA

730 ELE $=E L E+D I F L$

$\operatorname{TYP}(1, I$ TOTSA $)=O C 1$

$T Y P(2, I T O T S A)=0 C 2$

GO TO 30

$830 E L C=E L C+D I F L$

$T Y P(I, I T O T S A)=C B I$

TYP $(2, I T O T S A)=C B 2$

GO TO 30

$930 E L O=E L O+D I F L$

$\operatorname{TYP}(1, I T O T S A)=O B 1$

$\operatorname{TYP}(2, I T O T S A)=O B 2$

GO TO 30

330 WRITE $(3,2 \cup 3)$

$43 \cup$ CALL EXIT

$\begin{array}{llll}C & \text { RHOMOD IS MODERATOR DENSITY } \\ C & \text { RHOBOT IS BOAT } & \text { DENSITY }\end{array}$

$(G M / C C)$

0001830

0001840

0001850

0001860

0001870

0001880

0001890

0001900

0001910

0001920

0001930

0001940

0001950

0001960

0001970

0001980

0001990

0002000

0002010

0002020

0002030

0002040

$(G M / C C)$

0002050

$0 C 02060$ RHOSAM IS SAMPLE DENSITY

$(G M / C C)$

31 READ $(2,9)$ RHOMOD, RHOBOT, RHOSAM

0002070

0002080

0002090

WRITE $(3,1)$ DENT, RKEY,DCHD 1, DCHD2, CHNO, DDCHD 1, DOCHD2, OOTNO

0002100

WRITE $(3,2 \cup 4) X Z E R O,(T Y P(I, I), T Y P(2, I), X M I(I), I=1, I$ TOTSA)

WRITE $(3,2 \cup 5) N C, C E, N A, D E, N B, D P T, O D B, S P A C E, D O B I, T O X, D S$

$I C A N=C$

GO TO 1010

1011 ICAN $=1$

C AE - S ARE ACTIVATION ENERGIES

C FFC - $S$ ARE FREGUENCY FACTORS

$C$ IFA - $S$ ARE INIHIOITION FACTURS

IUIU READ 12,9$)$ H2OAE I, BIFFC1, H2OAE2, BIFFC2,BIIFA

READ $(2,9)$ CU2AE I, CIFFC1, CO2AE2, CIFFC2, CIIFA

C E - S ARE ACTIVATION ENERGIES

READ $(2,9) H 2 O E I, H 2 O L 2, H 2 O E 3$

$\operatorname{READ}(2,9) C O 2 E 1, \mathrm{CO} 2 E 2, \mathrm{CO} 2 E 3$

$C \quad K-S$ ARE FREQUENCY FACTORS

C K1O $(0 / 0 / K O D / M M H G)$

C K2C,K30 (I/MMHG)

C $2 O, A B$ AKE DIFFUSION FACTORS

C ZU (CM**2/(SEC*DEG**AB))

$C$ AD DIMLNSIONLESS

READ $(2,9) \mathrm{H} 2 \mathrm{OK} 10, \mathrm{H} 2 \mathrm{OK} 20, \mathrm{H} 2 \mathrm{OK} 30, \mathrm{ZOH} 2 \mathrm{O}$, ABH $2 \mathrm{O}$

READ $(2,9) C O 2 K 10, C O 2 K 20, C O 2 K 30,20 C O 2, A B C O 2$

READ $(2,9) \mathrm{GE}, \mathrm{DELE}, X K R A D, A C O N$

REAU $(2,9)$ RGE,RDELE,RACON

READ $(2,9) 02 \mathrm{~K} 10,02 E 1, A P W, 2002, A B O 2$

0002110

0002120

0002130

0002140

0002150

0002160

0002170

0002180

0002190

0002200

0002210

0002220

0002230

0002240

0002250

0002260

0002270

0002280

0002290

0002300

0002310

0002320

READ $(2,9) 02 K 20,02 E 2, B P W$

0002330

0002340

RMT = GAS TEMP (CENTIGRADE)

0002350 










$W G A M=A M * R H O M O D$

0003540

$W G A B=A B * R H O B O T$

0003550

WGAS $=A S *$ RHOSAM

0003560

PARTVC $=C N+(A N * D A 4+B N * U B T 4) / D C 4$

0003570

53U READ (2,9)PH2O5,PCO5,PCO25,PH25, VI,PO25

0003580

$I F(P H 205+P C O 5+P C O 25+P H 25+V I+P 025,1000,1000,630 \quad 0003590$

1000 IF(IEXIT) IUU, 100,1011

630 PH2O(5) $=$ PH2O5

$P C O(5)=P C O 5$

$P C O 2(5)=P C O 25$

0003600

0003610

0003620

$P 02(5)=P O 25$

$\mathrm{PH} 2(5)=\mathrm{PH} 25$

JOPH2 $=0$

$J O O X Y=0$

$J O C O=0$

JOHYD $=0$

$\mathrm{JOSH} 2 \mathrm{O}=0$

$\mathrm{JOSCO} 2=0$

$\mathrm{JOCO} 2=v$

$I Q=I$

$J Q=1$

$K Q=1$

$I J K=0$

0003630

0003640

0003650

0003660

0003670

0003680

0003690

0003700

0003710

0003720

0003730

0003740

0003750

0003760

CONST $=$ PH $25+P H 2 O 5$

C CONVERT CU FT PER MIN TO CC PER SEC

0003770 $V T=V I * 471.9502$

C FLOW VOLUMES

$\checkmark C=V T / P A R T V C$

$P \vee C=V C / D C 4$

$\vee B=P \vee C * D B T 4$

$\vee A=P \vee C * D A 4$

C VOLUME OUTSIDE CLOSED BOAT

$V C B O=V B / 2$

C VOLUME OUTSIDE OPEN BOAT

$V B O=V B / D O B 4 * D C E O 4 / 2$.

C VOLUME INSIDE OPEN BOAT

$V B I=V B O / D C B O 4 * D B I 4$

VOBT $=$ VBO+VBI

$C$ FACORS USED IN DERI $V$

C OPEN BOAT

VASO $=V B I / W G A S * P C K O U$

$V A B O=V O B T / W G A B * P C K O D$

$V A M O=V B O / W G A M * P C K O D$

C CLOSED BOAT

$V A B C=V C B O /(W G A S+N G A O) * P C K O D$

$V A M_{C} C=V C b O / W G A V_{1} * P C K O U$

C EMPTY CHANNEL

VAME $=$ VD/WGAM:

C FLOW PERCENTAGES

$V P C=1 C 0 . / V T$

VAOVT $=V A * V P C * A N$

$\vee B O V T=V \square * V P C * B N$

$V C O V T=V C * V P C * C N$

ASSIGN 440 TO IGO

ASSIGN 244 TO IAM

$X(b)=X Z E R O$

$Y I=X Z E R O$

CALL TIYI)

LINE $=12$

0003780

0003790

$0 C 03800$

0003810

0003820

0003830

0003840

0003850

0003860

0003870

0003880

0003890

0003900

0003910

0003920

0003930

0003940

0003950

0003960

0003970

0003980

0003990

0004000

0004010

0004020

0004030

0004040

0004050

0004060

0004070

0004080

0004090

0004100

0004110

0004120 
WRITE $(3,200)$ DENT

0004130

WRITE $(3,2 \cup 1)$ VCOVT, VAOVT, VBOVT

0004140

WRITE $(3,2 \cup 8)$ VI, PH 205, PCO5,PCO 25, PH 25, PO 25

0004150

WRITE $(3,202)$

$\operatorname{TYPE}(1)=\operatorname{TYP}(1,1)$

$\operatorname{TYPE}(2)=\operatorname{TYP}(2,1)$

$W I=Y I / C F I C M$

OWRITE $(3,4)$ TYPE, WI, PH2O(5), PCO (5), PH2 (5), PCO2(5), POZ (5), TYI

0004160

0004170

0004180

0004190

0004200

0004210

DO 24 INSA $=1$, ITOTSA

KOUT $=U$

NSAMPL $=N S A I$ I INSA)

$X N=X M I(I N S A) * C F I C M$

$D X D D=D X D D I(I N S A)$

ASSIGN 48 TO IPC

DATA QUU8CT/OUOUCU0000000/

DATA Q0O9CT/O777777777777/

IF(BOOL (OR (AND (DXDD,Q008CT), AND(COMPL(DXDD),Q009CT)))) 43,44,43

$C$

DXDU CHANGEU FOR THIS INTERVAL

$43 D D X D=D X D D * C F I C M$

44 ISTEP $=U$

$X_{0}=X(5)$

NOSTEP $=(X N-X C) / D D X D+.5$

344 DELX $=(X N-X O) / F L O A T(N O S T E P)$

GO TO IAM, $(144,244)$

144 IF (ABS( (DELX-DELTAX)/DELTAX)-.5E-7) $46,46,45$

244 IF(NOSTEP-4) $145,444,444$

444 ASSIGN 144 TO IAM

GO TO 245

C INTERVAL OF INTEGRATION CHANGED

45 IF (NOSTEP-4) $145,245,245$

145 NOSTEP $=4$

GO TO 344

245 DELTAX $=$ DELX

DX0304 =DELTAX $/ .75$

$D \times 02=D E L T A X / 2$.

$D \times 3=D E L T A X * 3$

$X(1)=X(5)$

PH2O(1) $=$ PH2O(5)

$P C O(1)=P C O(5)$

$P C O 2(1)=P C O 2(5)$

$P 02(1)=P 02(5)$

$\mathrm{PH} 2(1)=\mathrm{PH} 2(5)$

DRVH2O(1) =DRVH2O(5)

DRVCO $(1)=$ DRVCO $(5)$

DRVCO2(I) =DRVCO2(5)

2000 DRVO2 $(1)=$ DRVO2 $(5)$

ASSIGN 240 TO IWENT

GO TO 48

C INTERVAL OF INTEGRATION REMAINS THE SAME

46 ASSIGN 210 TO IWENT

146 DO $47 \quad I=1,4$

$X(I)=X(I+1)$

PH2O(I) $=$ PH2O(I+I)

$P C O(I)=P C O(I+1)$

$P C O 2(I)=P C O 2(I+1)$

$\mathrm{PO} 2(1)=\mathrm{PO} 2(\mathrm{I}+1)$

$\mathrm{PH} 2(\mathrm{I})=\mathrm{PH} 2(\mathrm{I}+1)$

DRVH2O(I) =DRVH $2 O(1+1)$

0004220

0004230

0004240

0004250

0004260

0004270

0004280

0004290

0004300

0004310

0004320

0004330

0004340

0004350

0004360

0004370

0004380

0004390

0004400

0004410

0004420

0004430

0004440

0004450

0004460

0004470

0004480

0004490

0004500

$0 C 04510$

0004520

0004530

0004540

0004550

0004560

0004570

0004580

0004590

0004600

0004610

0004620

0004630

0004640

0004650

0004660

0004670

0004680

0004690

0004700

$D R V C O(I)=D R V C O(I+1)$ 
$\operatorname{DRVCO} 2(I)=\operatorname{DRVCO} 2(I+1)$

0004720

2001 DRVO2 $(I)=\operatorname{DRVO} 2(I+1)$

47 CONTINUE

GO TO IPC, $(210,48)$

C

48 GO TO $(2 U, 21,22$, NSAMPL EMPTY CHANNEL

$20 \quad A V C=V A M E$

TYPE $(1)=O C 1$

$\operatorname{TYPE}(2)=O C 2$

C CLOSED DOAT

$21 \quad A \vee B=\vee A B C$

$A V C=\vee A M C$

TYPE $(1)=C B 1$

TYPE $(2)=C B 2$

GO TO 40

C

OPEN BOAT.

$22 \quad A V C=V A M O$

$A \vee B=\vee A B O$

$A V S=V A S O$

TYPE $(1)=O B 1$

$\operatorname{TYPE}(2)=O B 2$

40 GO TO IGO, $(340,440)$

340 GO TO IWENT, $(240,210)$

c

440 A.SSIGN 340 TO IGO

USE RUNGES METHOD TO GET STARTED

$Y I=X(1)$

CALL T(YI)

$K R K=4$

CALL DERIV(I)

240 CONT I NUE

AKRK $4=$ DELTAX*(DRVH2O( 1),

ALRK4 $\quad=$ DELTAX*(DRVCO $(1)$,

AMRK $4 \quad=$ DELTAX*(DRVCO2 $(1)$,

$2 \cup 02$ ANRK 4

$=$ DELTAX*( DRVOZ2(1))

140 KOUNT $=1$

DO $101=1,3$

I STEP $=$ I STEP +1

AKRK $1=A K R K 4$

$A L R K 1=A L R K 4$

AMRK $1=A M R K 4$

ANRK $1=$ ANRK 4

$K R K=1$

$Y I=Y I+D X 02$

CALL T(YI)

CALL DERIV $(I+1)$

$A K R K 2=D E L T A X * D R V H 2 O(I+1)$

$A L R K 2=D E L T A X * D R V C O(I+1)$

AMRK2 $=D E L T A X * D R V C O 2(I+1)$

$20 \cup 3$ ANRK $2=0 E L T A X * D R V O 2(I+1)$

$K R K=2$

CALL DERIV $(I+1)$

AKRK $3=U E L T A X * D R V H 2 O(I+1)$

$A L R K 3=D L L T A X * D R V C O(I+I)$

AMRK $3=D E L T A X * D R V C O 2(I+1)$

$2 \cup \cup 4$ ANRK $3=D E L T A X * D R V O 2(I+I)$

$Y I=Y I+D X 02$

CALL T(YI)

$K R K=3$

0004730

0004740

0004750

0004760

0004770

0004780

0004790

0004800

0004810

0004820

$0 C 04830$

0004840

0004850

0004860

0004870

0004880

0004890

0004900

0004910

0004920

0004930

0004940

0004950

0004960

0004970

0004980

0004990

0005000

0005010

0005020

0005030

0005040

0005050

0005060

0005070

0005080

0005090

0005100

0005110

0005120

0005130

0005140

0005150

0005160

0005170

0005180

0005190

0005200

0005210

$0 C 05220$

0005230

0005240

0005250

0005260

0005270

0005280

0005290

0005300

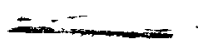


CALL DERIV $(I+1)$

0005310

$A K R K 4=D E L T A X * D R V H 2 O(I+1)$

0005320

$A L R K 4=D E L T A X * D R \vee C O(I+1)$

0005330

AMRK $4=D E L T A X * D R V C O 2(I+1)$

0005340

ANRK4 $=$ DELTAX*DRVO2 $(I+1)$

$P H 2 O(1+1)=P H 2 O(I)+(A K R K 1+2 * *(A K R K 2+A K R K 3)+A K R K 4) / 6$.

0005350

$P C O(I+1)=P C O(I)+(A L R K 1+2 * *(A L R K 2+A L R K 3)+A L R K 4) / 6$.

0005360

$P C O 2(1+1)=P C O 2(I)+(A M R K I+2 * *(A M R K 2+A M R K 3)+A M R K 4) / 6$.

0005370

0005380

0005390

0005400

$P O 2(I+1)=A M A X 1(P O 2(I+1), 0.1)$

PHZ $(I+1)=$ CONST $-P H 2 O(I+1)$

$X(I+1)=Y I$

IF(KOUNT-KRITE) $1 U, 110,110$

0005410

0005420

0005430

0005440

0005450

IF (LINE-MAXLIN) $410,310,310$

0005460

0005470

0005480

0005490

0005500

0005510

WRITE $(3,208) V I, P H 205, P C O 5, P C O 25, P H 25, P O 25$

WRITE $(3,202)$

CALCULATE BURNOUT RATES

$K R K=4$

CALL DERIV $(I+1)$

0005520

0005530

$W I=Y I / C F I C M$

$0 C 05540$

WRITE $(3,4)$ TYPE, WI, PH20(I+1),PCO(I+1),PH2(I+1), PCO2(I+1),PO2(I+1)0005550

$1, T Y I, B O R C, B O R B, B O R S$

$I J K=1 \mathrm{JK}+1$

$E H 2 O C H(I J K)=E H 2 O 1$

$E H 2 O S A(I J K)=E H 2 O 2$

$E(O 2 C H(I J K)=E C O 21$

$E C O 2 S A(I J K)=E C O 22$

$E O 2 S A(I J K)=E 022$

$H 2 O L P(I J K)=H 2 O L$

$C O 2 L P(I J K)=C 02 L$

$02 L P R(I J K)=02 L$

PRTYPI(IJK) = TYPE (1)

0005560

0005570

0005580

0005590

0005600

0005610

0005620

0005630

0005640

0005650

0005660

PRTYP 2 (IJK) = TYPE (2)

0005670

0005680

0005690

$W I P(I J K)=W I$

0005700

$K R K=4$

ASSIGN 2 IU TO IPC

0005710

0005720

$210 \quad I=1$

I STEP = I STEP+ I

$X(I+4)=X(I+3)+$ DELTAX

C PREUICTOR - CORRECTOR FORMULAE

C SEE RALSTON AND WILF, MATH METHOOS FOR DIGITAL COMP. PP 99-100

MILNES PREDICTOR FORMULA

$\mathrm{PH} 2 \mathrm{O}(\mathrm{I}+4)=\mathrm{PH} 2 \mathrm{O}(\mathrm{I})+$

$1(I+2)+2.0 * D R V H 2 O(I+3))$

PCO $(I+4)=$ PCO $(I)+$

$1(\mathrm{I}+2)+2.0 *$ ORVCO $(1+3) 1$

PCO2 (I+4) = PCO2(I) +

DXO3O4*(2.0*DRVH2O( I+1) - DRVH2O

0005730

0005740

0005750

0005760

0005770

0005780

0005790

0005800

0005810

0005820

$1(1+2)+2.0 *$ DRVCO2 $(1+3)$

DX0304*( 2.0*DRVCO (I+1) - DRVCO

$0 \times 0304 *(2.0 * D R V C O 21 I+1)-0 R V C O 2$

PO2 $(I+4)=P 02(I)+0 \times 0304 *(2.0 * D R V O 2(I+1)-D R V O 2(I+2)+$

0005830

0005840

0005850

0005860

0005870

PO2 $(I+4)=$ AMAXI $(P O 2(I+4) \cdot 0.1$

PH $2(I+4)=$ CONST - PH $2 O(I+4)$

0005880

$Y I=X(1+4)$

0005890 
CALL T(YI)

0005900

$N=1$

C CORRECTOR FORMULAE

0005910

HAMMINGS METHOD

15 CONT INUE

CALL DERIV(I+4)

PH2OA $=(9 . * P H 2 O(I+3)-P H 2 O(I+1)+D \times 3 *(D R V H 2 O(I+4)+2 . * D R V H 2 O(I+3)-$ IDRVH2O(I +2$)) 1 / 8$.

$P C O A=19 . \approx P C O \quad(I+3)-P C O(I+1)+D \times 3 *(D R V C O(I+4)+2 *$ DRVCO $(I+3)-$ IORVCO $(I+2)) / 8$.

$P C O 2 A=(5 . * P C O 2(I+3)-P C O Z(I+1)+D \times 3 *(D R V C O 2(I+4)+2 * * D R V C O 2(I+3)-$ 10RVCO2 $(I+2))) / 8$.

$\mathrm{PH} 2(\mathrm{I}+4)=\mathrm{CONST}-\mathrm{PH} 2 \mathrm{O} \dot{\mathrm{A}}$

2006 PO2A $=\left(9 . * P O 2(I+3)-P O_{2}(I+1)+D X_{3} *(D R V O 2(I+4)+2\right.$. DRVO $2(I+3)-$

1DRVO2 $(I+2)) 18$.

PO2A $=A M_{1} A X_{1}\left(P O 2 A,{ }^{\prime}\right)$

IFI $N-2112,14,14$

$12 N=N+1$

$P H 2 O(I+4)=P H 2 O A$

$P C O \quad(I+4)=P C O A$

$P C O 2(I+4)=P C O 2 A$

2007 PO2 $(I+4)=$ PO2A

C TEST TO haLVE THE INTERVAL SIZE

IF $(N-5) 15,15,301$

301 DDXD $=$ DDXO/2.

KOUT $=$ KOUT +1

$X(5)=X(4)$

$\mathrm{PH} 2 \mathrm{O}(5)=\mathrm{PH} 2 \mathrm{O}(4)$

$P C O(5)=P C O(4)$

$P \operatorname{CO} 2(1)=P \operatorname{CO} 2(4)$

$P 02(5)=P O 2(4)$

PH2 (5) $=$ PH2 $(4)$

DRVH2O(5) $=$ DRVH2O(4)

DRVCO $(5)=$ DRVCO (4)

DRVCO2 $(5)=$ DRVCO2 $(4)$

DRVO2 $(5)=$ DRVO2 $(4)$

GO TO 44

14 TEST $1=(\mathrm{PH} 2 \mathrm{CA}$

TEST $2=1$ PCO A

- PH2O(I I 4 ) /PH2OA

TEST $3=1 P C O 2 A$

- PCO I I+4) 1/PCOA

- PCO21 I+4 1)/PCO2A

$2 \cup \cup 8$ TEST $4=(P O 2 A-P O 2(I+4)) / P O 2 A$

IF( ABS( TEST1) - .5E-7) $16,16,12$

16 IF( $A B S($ TEST2, - .5E-7) 17,17, I2

17 IF( ABS( TEST3) - .5E-7) 18,18,12

18 IF ( ABS(TEST4) - .5E-7) 19,19,12

$19 \mathrm{PH} 2 \mathrm{O}(\mathrm{I}+4)=\mathrm{PH} 2 \mathrm{OA}$

$P C O(I+4)=P C O A$

$P C O 2(I+4)=P C O 2 A$

$2009 \mathrm{PO} 2(I+4)=P O 2 A$

IFI KOUNT - KRITE $141,42,42$

42 KOUNT $=U$

IF(LINE-MAXLIN) $242,142,142$

142 LINE $=8$

WRITE $(3,20)$ DENT

WRITE $(3,2 \mathrm{C} 8) \mathrm{VI}, \mathrm{PH} 205, \mathrm{PCO}, \mathrm{PCO} 25, \mathrm{PH} 25, \mathrm{PO} 25$

WRITE $(3,202)$

242 LINE $=L I N E+1$

$W I=Y I / C F I C M$

0005920

0005930

0005940

0005950

0005960

0005970

0005980

0005990

0006000

0006010

0006020

0006030

0006040

0006050

0006060

0006070

0006080

0006090

0006100

0006110

0006120

0006130

0006140

0006150

0006160

0006170

0006180

0006190

0006200

0006210

0006220

0006230

0006240

0006250

0006260

0006270

0006280

0006290

0006300

0006310

0006320

0006330

0006340

$00 \cup 6350$

0006360

0006370

0006380

0006390

0006400

0006410

oor 6420

0006430

0006440

0006450

0066460

0006470

OwRITE $(3,4)$ TYPE, I , PH2O(5), PCO(5), PH2 (5), PCO2(5), PO2(5), TYI, BORC, BOOO6480 
IORB, BORS

0006490

WRITE $(3,3)$ KOUT, N

0006500

$I J K=I J K+I$

$E H 2 O C H(I J K)=E H 2 O I$

0006510

$E H 2 O S A(I J K)=E H 2 O 2$

$E C O 2 C H(I J K)=E C O 2 I$

0006520

$E C O 2 S A(I J K)=E C O 22$

0006530

$E O 2 C H(I J K)=E 021$

$E 02 S A(I J K)=E 022$

$H 2 O L P(I J K)=H 2 O L$

$\operatorname{CO} 2 L P(I J K)=C O 2 L$

$02 L P R(I J K)=02 L$

PRTYPI (IJK) = TYPE ( 1$)$

0006540

0006550

0006560

0006570

0006580

0006590

PRTYP2 (IJK) $=$ TYPE (2)

0006600

TYIPR (IJK) = TYI

0006610

0006620

0006630

$W I P(I J K)=W I$

0006640

0006650

41 KOUNT $=$ KOUNT +1

IF (I STEP-NOSTEP) $146,141,14$ I

0006660

0006670

141 IF (KOUNT-1) $42,241,42$

0006680

JOKE $=J O P H L+J O O X Y+J O C O+J O H Y D+J O S H 2 O+J 0 S C O 2+J 0 C O 2$

0006690

IF ( JOKE) $3 \cup 2,302,3 \cup 4$

304 WRITE $(3,216)$ JOPH2, JUOXY, JOCU, JOHYD, JOSH 20, JOSCU2, JOCO 2

0006700

0006710

0006720

IF (IETA) $251,251,243$

243 NCOUNT $=$ I JK

0006730

UWRITE $(3,214)$ (PRTYPI(IX), PRTYP2 (IX), WIP(IX), EHZOCH(IX), EHZOSA (IX)0006740 I, ECO2CH(IX),ECO2SA (IX),EO2CH(IX), EO2SA (IX),H2OLP(IX), CO2LP(IX), O2LOO06750 2PR(IX), TYIPR (IX), I X = I, NCOUNT)

251 IF(IPLOT) $1 \cup 20,1020,1030$

1030 CALL GOPLOT

1020 GO TO 530

END

0006760

0006770

0006780

0006790

$0006800-$ 
COIO SUBROUTINE DERIVIJ)

SUBROUTINE DERIVIJ)

C THIS ROUTINE CALCULATES THE DERIVATIVES AT NODE I

DIMENSION PO2(5),DRVO2(5)

DIMENSION AI $(5,3), B I(5), C I(5), D I(5,3), X(5)$, DRVH $2 O(5)$, DRVCO(5),

1DRVCO2 (5), PH2O(5), PCO (5), PCO2 (5), PH2 (5),

2DENT( 12$)$, TYPE( 2$)$, NSAI $(50)$, XMI (50), DXDDI $(50), T Y P(2,50)$

COMMON $20 \mathrm{H} 2 \mathrm{O}$, ABH2O, NSAMPL, ZOCO2, ABCO2, YI

COMMON $S$, EXT, VKOODC, VKODB, VKODS, AVS

COMMON AVC, AVB, TY, UGC, R, RE

COMMON RD, RB, RS, H2OK10, H2OK2O, H2OK3O

COMMON H2OXI, H2OX2, H2OX3, CO2K1O, CO2K2O, CO2K3O

COMMON CO2XI, $C O 2 \times 2, \operatorname{CO} 2 \times 3$, TA, TB, AKRK1

COMMON AKRK2, AKRK3, AKRK4, ALRK1, ALRK2, ALRK3

COMMON ALRK4, AMRKI, AMRK2, AMRK3, AMRK4, KRK

COMMON DRVH2O, DRVCO

COMMON

COMMON

$P C O 2, A 1$
BIEX2, CIEXI

COMMON CIFFC2, BIIFA

COMMON

COMIMON

VB,

- Bl 1 , Cl, DI, BlExi

- Ciex2

- CIIFA

- Y

BIFFCl, BIFFC2, ClFFCl

- HALFVE, VCBO, RBT

, TYI, TKV , TK

COMMON

COMMION

$\mathrm{ZCO} 2, \mathrm{ZH} 2 \mathrm{O}$

- BICON

- DENS

DENB , RI

- R3

- CICONI, DENM

- EH2OI, EH2O2

COMMON

ECO21, ECO22

- ECO23

, NSAI , XMI

, EH2O3

H2OK1, H2OK2

- H2OK3

- NSAI

- DXDDI

- $\operatorname{CO} 2 \mathrm{~K} 3$

COMMON

COMMON

COMMON

DENT

- TYPE

- TYP

- RBSQ

- CO2K2

- ZCTK

$A M$, $A B$, AS

- RDSQ

COMMON

PCKOD, TIN

- TMAX

- RATIOL , EH2OT

- BORC

- BORB

- ECO2T

- BORS

COMMON

COMMON

ANRK 3

- COZL

- H2OKV

, CO2KV

, $\mathrm{PO} 2$

- ANRKI

, ANRK2

- O2L

- O2K1

, O2K2

COMMON

EO22

- EO2T

- O2KV

- $\mathrm{ABO} 2$

- E021

- EO23

, $O_{2} X_{1}$

COMMON

$02 \times 2$

- O2K10

, O2K20

, $G E$

- 2002

- XKRAD

, VFACT, TEFAC, WWA

COMMON ECAREA, CBAREA, OBAREA, TIK

- RGE

- RDELE

0100000

0100010

0100020

0100030

0100040

0100050

0100060

0100070

0100080

0100090

0100100

0100110

0100120

0100130

0100140

0100150

0100160

0100170

0100180

0100190

0100200

0100210

0100220

0100230

0100240

0100250

0100260

0100270

0100280

0100290

0100300

0100310

0100320

0100330

COMMON RACON, RGVAL, CICON2, WI

0100340

COMIMON /ANIV/CFICV, IQ, JO, KQ, RUUCH(450), RQOOS(450), RQOCS(450), DQOCH(0100350
$145 U)$, DQOOS(453),DQOCS(450)

COMVION / JAN/ EH2OCH(450), EH2OSA(450), ECO2CH(450), ECO2SA(450), EO2CHO100370

$X(450), E O 2 S A(450), H 2 O L P(450), C O 2 L P(450), O 2 L P R(450)$, PRTYPI (450), PRTYO 100380 $X P 2(450)$, TYIPR $(450)$, WIP $(450)$

COMIMON / JUKES/ JOPH2, JOOXY, JOCO, JOHYO, JOSH2O, JOSCO2, JOCO2

0100390

$I=J$

GO TO $(5,6,7,4), K R K$

0100400

$4 \mathrm{PH} 2 \mathrm{OI}=\mathrm{PH} 2 \mathrm{O}(1)$

$P H 2 I=P H 2(I)$

$P C O I=P C O(I)$

$P C O 2 I=P C O 2(I)$

PO2I = PO2 (I)

GO TO 8

5 AKO2 $=$ AKRK $1 / 2$

$\mathrm{PH} 2 \mathrm{OI}=\mathrm{PH} 2 \mathrm{O}(\mathrm{I}-1)+\mathrm{AKO} 2$

$P H 2 I=P H 2(I-1)-A K O 2$

0100410

0100420

0100430

0100440

0100450

0100460

0100470

0100480

0100490

0100500

$P C O I=P C O(1-1)+A L R K 1 / 2$.

$P C O 2 I=P(O 2(I-1)+A M R K I / 2$.

0100510

0100520

0100530

PO2I $=$ PO2 $(I-1)+A N R K 1 / 2$

0100540

GO TO 8

0100550

6 AKO2 = AKRK2 $/ 2$.

PH2OI $=$ PH2O I-1, $+A K O 2$

0100560

PHZI = PHZ II-1)-AKO2

0100570

0100580 
$P C O I=P C O(I-1)+A L R K 2 / 2$.

0100590

$P C O 21=P C O 2(I-1)+A M R K 2 / 2$.

0100600

PO2I $=$ PO2 $(I-I)+A N R K 2 / 2$.

0100610

GO TO 8

$7 \mathrm{AKO2}=\mathrm{AKRK} 3$

$\mathrm{PH} 2 \mathrm{OI}=\mathrm{PH} 2 \mathrm{O}(\mathrm{I}-\mathrm{I})+\mathrm{AKO} 2$

$P H 2 I=P H 2(I-1)-A K O 2$

$P C O I=P C O(I-1)+A L R K 3$

$P C O 2 I=P C O 2(I-1)+A M R K 3$

0100620

0100630

0100640

0100650

0100660

$P O 2 I=P O 2(I-1)+A N R K 3$

0100670

$\begin{array}{ll}C & T K=6.01019 E-7 \\ C & T K V=T K * T Y\end{array}$

C SEE SUBROUTINE T

$K I=K I \cup * E X P F\left(X_{1} / T\right)$

$K 2=K 20 * E X P F\left(X_{2} / T\right)$

$K 3=K 3 J * E X P F\left(X_{3} / T\right)$

0100680

0100690

0100700

0100710

0100720

0100730

0100740

SEE SUBROUTINE T

0100750

RH IS THE HOMOGENEOUS REACTION RATE

0100760

8 IF (PH2 I ) 82,82,84

0100770

0100780

$\mathrm{JOPH} 2=1$

GO TO 83

$84 \mathrm{PH} 2 \mathrm{IX}=\mathrm{PH} 2 \mathrm{I}$

$83 \mathrm{RHH} 2 \mathrm{O}=\mathrm{H} 2 \mathrm{OKI}$ (PH2OI/(I•+H2OK2*SQRT(PH2IX)+H2OK3*PH2OI)

0100790

0100800

0100810

0100820

$\mathrm{KH} \mathrm{CO} 2=\mathrm{CO} 2 \mathrm{KI} * \mathrm{PCO} 2 \mathrm{I} / 1 \mathrm{I} *+\mathrm{CO} 2 \mathrm{~K} 2 * \mathrm{PCOI}+\mathrm{CO} 2 \mathrm{~K} 3 * \mathrm{PCO} 2 \mathrm{I})$

0100830 IF $(02 K 1) 91,91,93$

93 IF(PO2I-.UUU76) $88,88,86$

88 JOOXY $=1$

$91 \mathrm{RHO2}=0$.

GO TO 89

$86 \mathrm{RHO} 2=02 \mathrm{~K} 1 * \mathrm{PO} 2 \mathrm{I} * * \mathrm{APW}$

$89 \mathrm{PKH} 2 \mathrm{O}=\mathrm{RHH} 2 \mathrm{O} * \mathrm{TKV}$

$P K C O 2=R H \quad C O 2 * T K V$

$P K O 2=$ RHO2 *TKV

$K V$ IS THE VOLUNE $\mathrm{H} 2 \mathrm{OKV}=\mathrm{PKH} 2 \mathrm{O} / \mathrm{PH} 2 \mathrm{OI}$

$\mathrm{CO} 2 \mathrm{KV}=\mathrm{PKCO} 2 / \mathrm{PCO} 2 \mathrm{I}$

$02 K V=P K O 2 / P O 2 I$

C $Z$ IS THE EFFECTIVE UIFFUSION COEFFICIENT

$C$ L IS THE DEPTH OF OXIDATION*SQRTF(GRAPHITE DENSITY)

0100840

0100850

0100860

0100870

0100880

0100890

0100900

0100910

0100920

0100930

0100940

0100950

0100960

0100970

0100980

0100990 $\mathrm{H} 2 \mathrm{OL}=S Q R T(Z \mathrm{H} 2 \mathrm{O} / \mathrm{H} 2 \mathrm{OKV})$ CO2L $=S Q R T(Z C O 2 / C O 2 K V)$

$O 2 L=S Q R T(Z O 2 / O 2 K V)$

C ETA CALCULATES THE DIFFUSION FACTOR

CALL ETAIH2OL, EH2O2, EH2O3, EH2O2, EH2OT)

CALL ETA (CO2L,ECO2I,ECO23,ECO22, ECO2T)

CALL ETA (O2L,EO21, EO23,EO22, EO2T)

$\begin{array}{ll}T \text { AI IS THE } & \mathrm{C}+\mathrm{H} 2 \mathrm{O} \\ T \text { DI IS THE } & \mathrm{C}+\mathrm{CO} 2 \\ \text { TFI IS THE } & \mathrm{C}+02 \\ \text { TF2 IS THE } & \mathrm{C}+\mathrm{O} 2\end{array}$

REACTION RATE TERIM

REACTION RATE TERM

REACTION RATE TERM

TF2 IS THE

REACTION RATE TERM (RADIATION)

0101000

0101010

0101020

0101030

0101040

0101050

0101060

0101070

0101080

0101090

0101100

0101110

0101120

$60 \quad$ TO 25

24 TF2 $=02 \mathrm{K2} * \mathrm{PO} 2 \mathrm{I} * \mathrm{BPW}$

$25 \mathrm{BORC}=-\left(\mathrm{EH} 2 \mathrm{O} 1 * \mathrm{RH} \mathrm{H}_{2} \mathrm{O}+\mathrm{ECO} 21 * \mathrm{RH} \mathrm{CO} 2+\mathrm{EO}_{2} 1 * \mathrm{RHO} 2+\mathrm{TF} 2\right)$

$R Q O C H(I Q)=B O R C$

DQOCH I IQ $)=Y I / C F I C M$

0101230

0101140

0101150

0101160

$I Q=I Q+I$

0101170 
$T A 1=E H 2 O 1 / A V C$

0101180

$T D 1=E C O 21 / A V C$

0101190

$T F 1=E O 21 / A V C$

0101200

IF (NSAMPL-2) $9,16,11$

0101210

EMPTY CHANNEL

$9 \mathrm{BORB}=0$.

BORS $=0$

GO TO 12

C

CLOSED BOAT

li $B O R B=-(E H 2 O 3 * R H H 2 O+E C O 23 * R H C O 2+E O 23 * R H O 2+T F 2)$

SORS $=-(E H 2 O 2 * R H H 2 O+E C O 22 * R H C O 2+E O 22 * R H O 2+T F 2)$

0101220

0101230

0101240

0101250

0101260

0101270

0101280

0101290

$D Q O C S(K Q)=Y I / C F I C M$

0101300

$K Q=K Q+1$

$T A l=T A 1+E H 2 O T / A V B$

0101310

$T D 1=T D 1+E C O 2 T / A V B$

$T F I=T F I+E O 2 T / A V B$

0101320

0101330

0101340

GO TO 12

c

OPEN EOAT

$11 \mathrm{BORB}=-(\mathrm{EH} 2 \mathrm{O} 3 * \mathrm{RH} \mathrm{H} 2 \mathrm{O}+\mathrm{ECO} 23 * \mathrm{RH} \mathrm{CO} 2+\mathrm{EO} 23 * \mathrm{RHO} 2+\mathrm{TF} 2)$

$B O R S=-(E H 2 O 2 * R H H 2 O+E C O 22 * R H C O 2+E O 22 * R H O 2+T F 2)$

0101350

0101360

0101370

RQOOS $(J Q)=$ IORS

DQOOS $(J Q)=Y I / C F I C M$

$J Q=J Q+1$

$T A 1=T A 1+E H 2 O 3 / A V B+E H 2 O 2 / A V S$

$T D 1=T D 1+E C O 23 / A V B+E C O 22 / A V S$

$T F 1=T F 1+E 023 / A V B+E 022 / A V S$

0101380

0101390

0101400

0101410

0101420

0101430

0101440

0101450

0101460

0101470

0101480

0101490

0101500

0101510

0101520

0101530

0101540

0101550

0101560

0101570

0101580

0101590

0101600

0101610

0101620

0101630

0101640

$\begin{aligned} 72 C 1(I) & =0.0 \\ \text { JOHYD } & =1\end{aligned}$

GOHYD $=$

$\begin{array}{rr}740 C 1(I)=C I C O N 1 * P C U 2 I * \text { SURT }(P H 2 I / T Y) / T Y+C I C O N 2 * P C O 2 I * 1(P H 2 I 0101650 \\ 1 / T Y) * 0.33333) / T Y & 0101660\end{array}$ $1 / T Y) * 0.33333) / T Y$

76 IFINSAMPL $-2,26,27,28$

0101670

EMPTY CHANNEL

$26 B I(I)=B I(I) * E(A R E A$

$C_{1}(I)=C I(I) * E C A R E A$

GO TO 29

C

CLOSED $\triangle O A T$

$27 B I(I)=B I(I) * C B A R E A$

0101680

0121690

0101700

0101710

0101720

$C_{1}(I)=C I(I) * C B A R E A$

0101730

0101740

GO TO 29

c

OPEN BOAT

0101750

0101760 


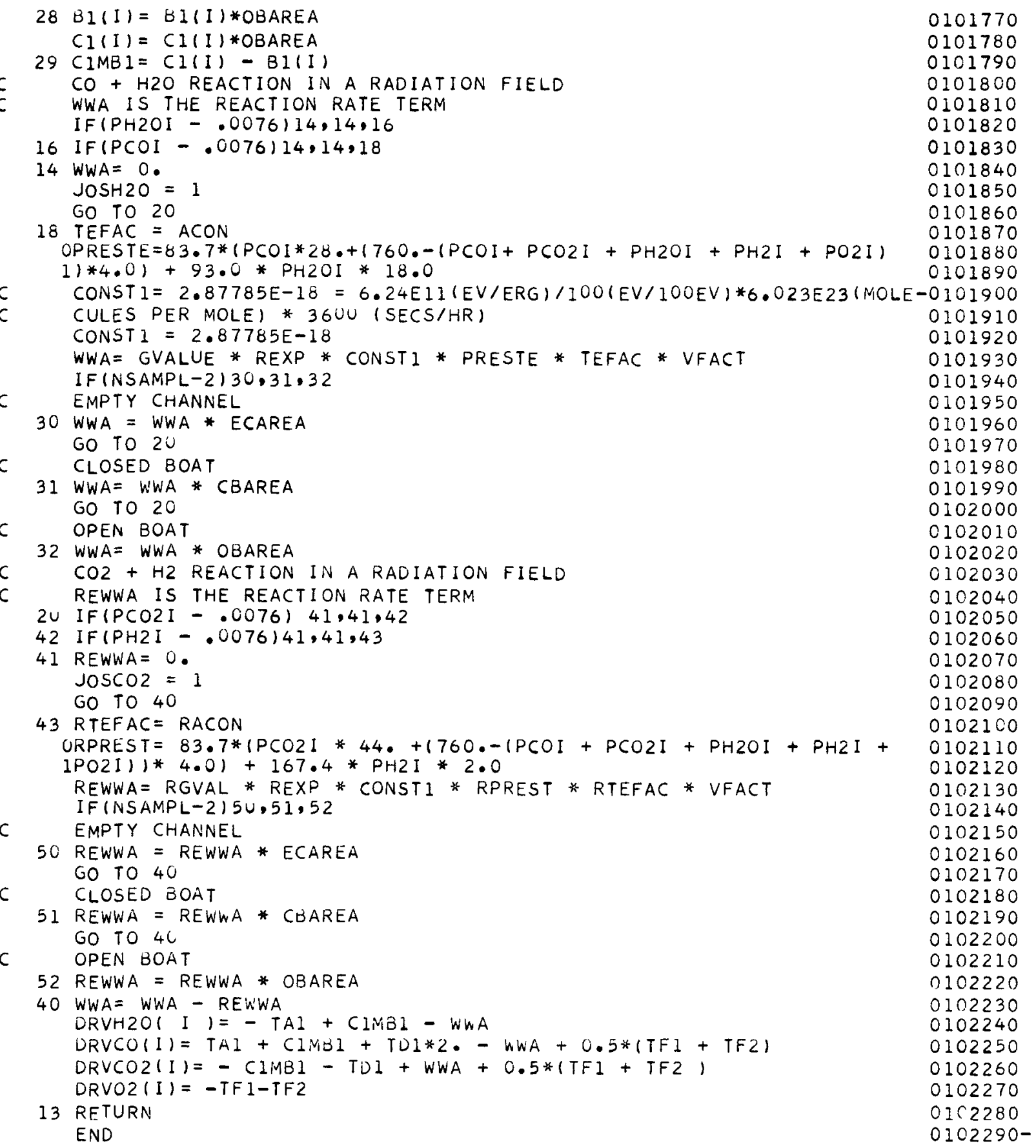


CU2O SUBROUTINE T(YI)

0200000

SUBROUTINE T(YI)

DIMENSION PO2 (5),DRVO2(5)

DIMENSION AI $(5,3), B I(5), C I(5), D I(5,3), X(5)$, DRVH2O(5), DRVCO(5),

0200010

0200020

IDRVCO2(5), PH2O(5), PCO (5), PCO2 (5), PH2 (5),

2DENT (12), TYPE (2), NSAI $(50), X M I(50)$, DXDDI $(50), T Y P(2,50)$

COMMON ZOHZO, ABHZO, NSAMPL, ZOCO2, ABCO2, YI

COMMON $S$, EXT, VKODC, VKODB, VKODS, AVS

COMMON AVC, AVB, TY, UGC, R, RE

COMMON RO, RB, RS, H2OK10, H2OK2O, H2OK3O

COMMON H2OX1, H2OX2, H2OX3, CO2K10, CO2K2O, CO2K3O

COMMON CO2X1, CO2X2, CO2X3, TA, TB, AKRKI

COMMON AKRK2, AKRK3, AKRK4, ALRK1, ALRK2, ALRK3

COMMON ALRK4, AMRK1, AMRK2, AMRK3, AMRK4, KRK

COMMON DRVH2O, DRVCO, DRVCO2, $\mathrm{PH} 2 \mathrm{O}, \mathrm{PH} 2, \mathrm{PCO}$

COMMON PCO2, AI, $21, \mathrm{~B} 1, \mathrm{Cl}, \mathrm{BIEXI}$

COMMON BIEX2, CIEXI, CIEX2, BIFFCI, BIFFC2, CIFFCI

COMMON CIFFC2, BIIFA

- CIIFA

COMMON

COMMON

$\mathrm{VB}, \mathrm{X}, \mathrm{Y}$

COMMON

$2 \mathrm{CO} 2,2 \mathrm{H} 2 \mathrm{O}$

- TYI

- TKV

- RBT

COMMON

DENB

, RI

- BICON

- CICONI

- TK

COMMON

ECO21

- R3

- $\mathrm{EH} 2 \mathrm{Ol}$, EH2O2

- DENS

COMMON
COMMON

H2OKI, H2OK2

- ECO23

- NSAI

- XiYI

- $\mathrm{EH} 203$

DENT

, TYPE

, H2OK3

- CO2KI

- COZK

, DXDOI

COMMON

AM

, $A B$

, TYP

- RBSQ

- RDSQ

, CO2K3

COMMON

PCKOD

- AS

- RATIOL, EH2OT

- ZCTK

COMMON

$\mathrm{H} 2 \mathrm{OL}$

- TIN

- TMAX

- BORC

- BORZ

- ECO2T

COMMON

ANRK 3

- CO2L

- H2OKV

- CO2KV

- BORS

COMMON

$A P W$

- ANRK4

- DRVO2

- $\mathrm{PO} 2$

- ANRKI

- ANRK2

COMMON

EO22

- BPW

- O2KV

- O2L

- O2K1

- O2K2

COMiviON

$\mathrm{O} 2 \times 2$

- EO2T

- 202

- $A B O 2$

- EO21

- E023

COMMON ECAREA, CBAREA, OBAREA, TIK

ACON

, O2KIO

- O2K20

- GE

, 2002

, O2X1

- gValue, ReXP

- VFACT, TEFAC

- XKRAD

- RGE

- WWA

$T Y I=T A+T B * S I N((2 \cdot * Y I+E X T) * S)$

C CONVERT TO KELVIN FOR COMPUTATION

$T Y=T Y I+Z C T K$

C

C SEE SUBROUTINE DERIV

$H_{2} O K I=H 2 O K 10$ *EXP( $\left.H_{2} O X 1 / T Y\right)$

$H_{2} O K 2=H 2 O K 2 O * \operatorname{EXP}\left(H_{2} O X 2 / T Y\right)$

$\mathrm{H} 2 \mathrm{OK} 3=\mathrm{H} 2 \mathrm{OK} 30 * \mathrm{EXP}(\mathrm{H} 2 \mathrm{O} \times 3 / T Y)$

CO2KI $=$ CO2KIO *EXP $($ CO2XI/TY $)$

CO2K2 $=$ CO2K2O *EXP( CO2X2/TY $)$

CO2K3 $=$ CO2K3U *EXP( CO2X3/TY)

$\mathrm{ZH} 2 \mathrm{O}=(\mathrm{ZOH} 2 \mathrm{O}) *(\mathrm{TY} * * \mathrm{ABH} 2 \mathrm{O})$

$Z C O 2=(20 C O 2) *(T Y \quad * * A B C O 2)$

C $\mathrm{CO}+\mathrm{H} 2 \mathrm{O}$ IN A GAMMA FIELD

GVALUE $=G E *$ EXP(+DELE $($ UGC*TY))

C $\mathrm{CO} 2+\mathrm{H} 2$ IN A RADIATION FIELD

RGVAL = RGE * EXP(RDELE/(UGC*TY))

REXP $=X K R H D * \operatorname{SIN}((2 . * Y I+E X T) * S)$

VFACT $=T I K /(V B * T Y$ )

$O_{2 K 1}=02 K 10 * \operatorname{EXP}(O 2 \times 1 / T Y)$

$02 K 2=02 K 20 * \operatorname{EXP}(O 2 \times 2 / T Y)$

$\mathrm{ZO2}=2002 *(T Y * * A B O 2)$

$1 T K V=T K * T Y$

BICON $=\left(B I F F C 1 * E X P\left(B I E X_{1} / T Y\right)+B 1 F F C 2 * E X P\left(B 1 E X_{2} / T Y\right)\right) /(V B * T Y)$

CICON $1=C I F F C I * E X P(C I E X I / T Y) * T I K / V B$

CICON $2=\operatorname{CIFFC2} * \operatorname{EXP}(C I E X 2 / T Y) * T I K / V B$

RETURN

NT PARTS OF THE EQUATIONS

0200030

0200040

0200050

0200060

0200070

0200080

0200090

0200100

0200110

0200120

0200130

0200140

0200150

0200160

0200170

0200180

0200190

0200200

0200210

0200220

0200230

0200240

0200250

0200260

0200270

0200280

$0<00290$

0200300

0200310

0200320

0200330

0200340

0200350

0200360

0200370

0200380

0200390

0200400

0200410

0200420

0200430

0200440

0200450

0200460

0200470

0200480

0200490

0200500

0200510

0200520

0200530

0200540

0200550

0200560

0200570

0200580

0200590

0200600

$0200610-$ 
C030 SUBROUTINE ETA (ALL, ETAM, ETAB, ETAS, ETABS)

SUBROUTINE ETA (ALL, ETAM, ETAB, ETAS, ETABS)

C THIS ROUTINE CALCULATES THE REACTION RATE FACTORS

0300000

0300010

C ALL IS THE DEPTH OF OXIDATION*SQRTF(GRAPHITE DENSITY)

0300020

ETAM IS DIFFUSION FACTOR FOR MODERATOR

ETAB IS DIFFUSION FACTOR FOR BOAT

ETAS IS DIFFUSION FACTOR FOR SAMPLE

DIMENSION PO2(5),DRVO2(5)

DIMENSION AI $(5,3), Z_{1}(5), C_{1}(5), D I(5,3), X(5), D R V H 2 O(5), D R V C O(5)$,

0300030

0300040

0300050

0300060

0300070

1DRVCO2 (5), PH2O(5), PCO (5), PCO2 (5), PHZ (5),

2DENT(12), TYPE (2), NSAI $(50)$, XMI $(50)$, DXDDI $(50), T Y P(2,50)$

COMMON ZUH2O, ABH2O, NSAMPL, ZOCO2, ABCO2, YI

COMMON AVC, AVB $:$ : TYODC, VKODB, VKODS, AVC,

COMMON RD, RB, RS, H2OK10, H2OK2O, H2OK3O

COMMON H2OXI, H2OX2, H2OX3, CO2K1O, CO2K2O, CO2K3O

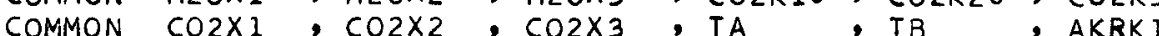

COMMON AKRK2, AKRK3, AKRK4, ALRK1, ALRK2, ALRK3

COMMON ALRK4, AMRK1, AMRK2, AMRK3, AMRK4, KRK

COMMON DRVH2O, DRVCO, DRVCO2, PH2O, PH2, PCO

COMMON PCO2, AI , Z1, 1 CI, DI, BIEXI

COMMON BIEX2, CIEXI, CIEX2, BIFFC1, BIFFC2, CIFFCI

COMMON CIFFC2, BIIFA, CIIFA, HALFVB, VCBO, RBT

COMMON VB, $X$, Y TYI, TKV, TK

COMMON ZCO2, ZHZO, BICON, CICONI, DENM, DENS

COMMON DENB, RI, R3, RH2OI, EH2O2, EH2O3

COMMON ECO21, ECO22, ECO23, NSAI, XMI, DXDDI

COMINON H2OKI, H2OK2, H2OK3, CO2KI, CO2K2, CO2K3

COMMON DENT, TYPE, TYP, RBSQ, RDSQ, ZCTK

COMMON AM, AB, AS, RATIOL, EHZOT, ECOZT

COMMON PCKOD, TIN, TMAX, BORC, BORB, BORS

COMMON HZOL, COZL, HZOKV, COZKV, ANRK1, ANRK2

COMMON ANRK3, ANRK4, DRVO2, PO2, O2K1, O2K2

COMMON APW, BPW, O2KV, O2L, EO2I, EO23

COMMON EO22, EO2T, ZO2, ABO2, ZOO2, O2XI

COMMON O2X2, O2K10, O2K20, GE, DELE, XKRAD

COMMON ACON, GVALUE, REXP, VFACT, TEFAC, WWA

COMMON ECAREA, CBAREA, OBAREA, TIK, RGE, RDELE

0300080

0300090

0300100

0300110

0300120

0300130

$0 \equiv 00140$

0300150

0300160

0300170

0300160

0300190

0300200

0300210

0300220

0300230

0300240

0300250

0300260

0300270

0300280

0300290

0300300

0300310

0300320

0300330

0300340

0300350

0300360

0300370

0300380

0300390

0300400

0300410

0300420

0300430

0300440

0300450

0300460

0300470

0300480

0300490

0300500

0300510

0300520

0300530

0300540

0300550

0300560

0300570

$2 S L=A L * D E N S$

$B L=A L * D E N B$

0300580

$X I=R S / S L$ 
$X_{2}=R B / B L$

0300590

$\times 3=R D / B L$

0300600

$B I O X I=B O(X 1)$

0300610

$B I \cup X 2=B O(\times 2)$

0300620

$B I O \times 3=B O(\times 3)$

0300630

$B I I X I=B I(X I)$

0300640

$B I 1 \times 2=B 1(\times 2)$

$B I 1 \times 3=B \perp(\times 3)$

0300650

0300660

0300670

$B K \times 2=O K(\times 2)$

0300680

$B K O \times 3=O K(\times 3)$

0300690

$B K 1 \times 3=5 K(\times 3)$

0300700

SAMR = BI $1 \times I / B I O X 1$

0300710

IF(NSAMPL-2) $3,3,4$

0300720

CLOSED BOAT CASE

0300730

3 RLSI I =RATIOL *BIIXI

0300740

0300750

$\begin{array}{ll}P S I=B I \cup \times 3 * S C R I P A+(B K O \times 3 * B I I \times 2) * B I O \times I-(B K O \times 3 * R L S I 1) * B I O \times 2 & 0300760 \\ \text { SCRIPC=BIUXI)(X2*PSI) } & 0300770\end{array}$

C BOAT

0300780

ET3 =2.*(RD*(SCRIPA*BI IX3-( (BKIX3*RIOXI)*BIIX2-(BKI)3*BIOX2)*0 0300790

IRLSI 1$))-R B *\left(S C R I P A * B I 1 \times 2-\left(\left(B K I \times 2 * B I O X_{1}\right) * B I 1 \times 2-(B K 1 \times 2 * B I O \times 2) * R L S 110300800\right.\right.$

2) ) / PSI

SAMR = SAMR*SCKIPC

C CALCULATE ETA FOR BOAT SAMPLE COMBINATION

$X_{6}=R B T / B L$

$B I 0 \times 6=B O(\times 6)$

$B I 1 \times 6=B I(\times 6)$

$E T A B S=2 . / \times 6 *(B I 1 \times 6 / 8 I 0 \times 6)$

GO TO 5

C OPEN BOAT CASE

BOAT

CASE

$4 E T 3=2 . *((B K O \times 3-B K O \times 2) /(B I O \times 2 * B K O \times 3-B I O \times 3 * B K C \times 2) *(R D * B I 1 \times 3-0$

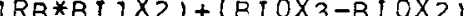
2BK $1 \times 211$

C R3=RD**2-RB* $* 2$

5 RUN3 $=$ R $3 / B L$

$E 3=E T 3 / R U N 3$

C SAMPLE

$E 2=2 \bullet / X 1 * S A M R$

CHANNEL

$6 \quad X 4=R E / C L$

$X 5=R \quad / C L$

BI $O \times 4=B O(\times 4)$

BI $1 \times 4=B 1(\times 4)$

$B I 1 \times 5=B 1(\times 5)$

$B K \cup \times 4=0 K(\times 4)$

BK $1 \times 4=5 K(\times 4)$

$B K 1 \times 5=S K(\times 5)$

ETI

$$
=
$$

2.

$B K O \times 411$

$E T A M_{1}=E T 1 / R U N 1$

$E T A S=E 2$

$E T A B=E 3$

9 RETURN

END

0300810

0300820

0300830

0300840

0300850

0300860

0300870

0300880

0300890

0300900

0200920

0300930

0300940

0300950

0300960

0300970

0300980

0300990

0301000

0301010

0301020

0301030

0301040

0301050

0301060

0301070

0301080

0301090

0301100

0301110

0301120

0301130

$0301140-$ 

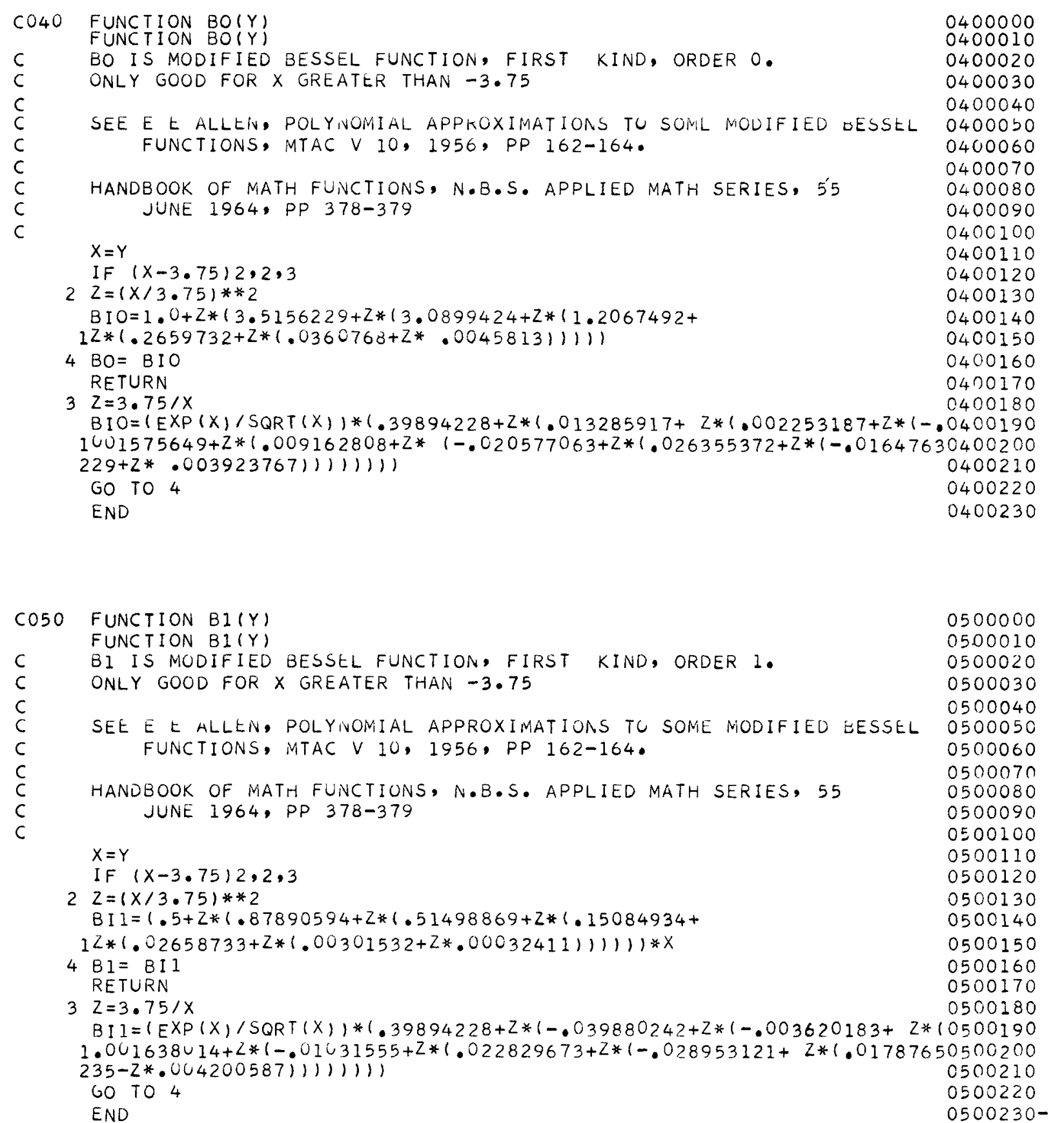


CO80 SUBROUTINE GOPLOT

0800000 SUBROUTINE GOPLOT

0800010

DIMENSION NY(3),XX(450), YY $(450,3)$, TEMP $(450), X T I T L E(19), Y T I T L E(8,200800020$

1), CHAR ( 5 )

0800030

INTEGER XTITLE, YTI TLE, CHAR

0800040

COMMON /A*N/CFICM,IQ, JQ,KQ,RQOCH (450), RQOOS(450), RQOCS(450), DQOCH $(0800050$

$1450), D Q O O S(453), 0 Q O C S(450)$

COMMON / JUDY/ Z(3600)

0800060

COMMON /BPLOTI/ YTITLE,XTITLE,CHAR

0800070

$N Y(1)=I Q-I$

$N Y(2)=J Q-1$

$N Y(3)=K Q-1$

$N X=I Q-1$

$K=0$

NOYST $=3$

$N_{1}=I Q-1$

$N 2=J Q-I$

$N 3=K Q-1$

$N Q=N 1+N 2+N 3$

$N_{4}=N Q+N 1$

$N 5=N 4+N 2$

IF $(N]) 2,2,4$

4 DO $100 \quad I=1, N 1$

0800080

0800090

0800100

0800110

0800120

0800130

0800140

0800150

0800160

0800170

0800180

0800190

0800200

0800210

$k=k+1$

0800220

o 000230

0800240

0800250

0800260

0800270

0800280

0800290

0800300

0800310

0800320

0800330

0800340

0800350

0800360

0800370

0800380

0800390

0800400

0800410

0800420

0800430

0800440

0800450

0800460

0800470

0800480

0800490

0800500

0800510

0800520

0800530

0800540

0800550

0800560

0800570

0800580

$0800590-$ END 
CO9O SUBROUTINE PLOT ( MODE, KRID, NUML, NX, NOYS, NY, XX, YY, TEMP ) SUBROUTINE PLOT ( MODE, KRID, NUML, NX, NOYS, NY, XX, YY, TEMP) PLOT SUBROUTINE REQUIRES MSORT AND LSORT SUBROUTINES.

ALSO A BLOCK DATA SUBROUTINE TO DEFINE OCTAL CONSTANTS PLUS AND BLANK.

THESE THREE ROUTINES ARE PRE-WRITTEN.

USER MUST WRITE MAIN CALLING PROGRAM AND HAS OPTION OF DEFINING TITLES AND PLOTTING SYMBOLS IN A BLOCK DATA PROGRAN OR READING THEM AS DATA.

USER SHOULD AUVANCL PRINTER IN MAIN PROGRAM AS PLOT WILL START ON THE NEXT LINE.

SPECIFICATION STATEMENTS

USER MUST MAINTAIN CORRESPONDENCE BETWEEN VARIABLE TYPES IN

MAIN AND SUB PROGRAMS.

LABELED COMMON BLOCKS MUST hAVE the sAME NAMES.

DIMENSION XX(NX), YY(NX,NOYS), NY(NOYS), TEMP(NX), IFULL(20), IYTITLE $(8,20), X T I T L E(30), C H A R(20)$,

2 YSCALE( 11$), S F Y(20), Y M I N(20), J S U M(20), L I N E(101)$

COMMON / JUDY/ Z(3600)

COMMON /BPLOTI/ YTITLE,XTITLE, CHAR

COMMON /BPLOT2/PLUS,BLANK

LOGICAL SLINE

INTEGER XTITLE, YTITLE, CHAR, PLUS, BLANK, XLINE

$c$

C INITIALIZE. ENSURE CONSISTENCY OF INPUT.

C MAKE A REASONABLE DECISION WHERE POSSIBLE TO ALLOW EXECUTION.

C IS MODE VALID.

100 IFI MODE.EQ.1 .OR. MODE.EQ.2.OR. MODE.EQ.3, GO TO 110 WRITE $(6.190)$ MODE RETURN

190 FORMATI 3 UHIPLOTTING MODE IS INVALID. - $1 /$ 3UH RETURN TO CALLING PROGRANI
A

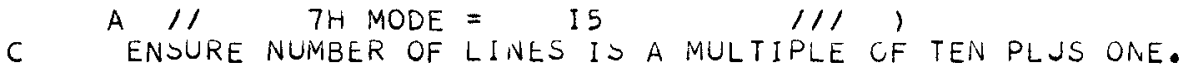

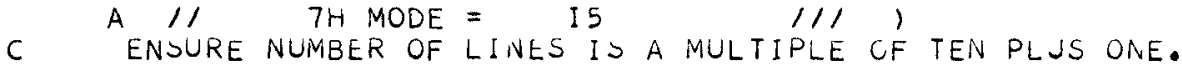

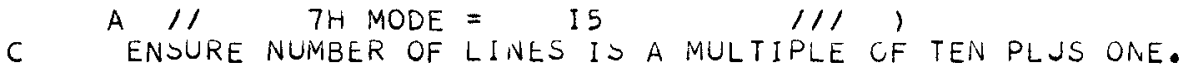

110 NUML $=1+10 *(1$ NUML $-1,1,10)$

C IS GRID STYLE VALID. IF NOT CHOOSE OPEN STYLE. IF ( NOT. ( KRID.EQ.I $\bullet O R \cdot K R I D \cdot E Q \cdot 2$, , KRID $=2$

C IF MODE $=1$ COMPLETE THOSE TAOLES MAIN PROGRAM IS ALLOWED TO

C IGNORE.

IF( MODE.NE.I I GO TO 200

DO $120 \mathrm{~J}=1$, NOYS

$N Y(J)=N X$

$120 \quad \operatorname{IFULL}(J)=J$

C SET UP AIDS FOR SEARCHING ARRAYS.

$C$ JSUM SERVES AS SUBSCRIPT ORIGIN AND IS SUM OF NUMBER OF

C PRECEDING ELEMENTS OF YY IN COLUMN ORDER. NYSUM IS TOTAL NUMBER

C OF POINTS TO BE PLOTTED.

200 JSUM(I) $=0$

DO $210 \mathrm{~J}=2$, NOYS

$210 \operatorname{JSUM}(J)=\operatorname{JSUM}(J-1)+\operatorname{NY}(J-1)$

NYSUM = JSUM(NOYS) + NY(NOYS)

C ORANCH TO SORTING ROUTINE APPROPRIATE TO FURM OF DATA.

IFI MODE - $2,221,222,223$

C MODE $=1$. ONE $X$ COLUMN, ALL Y COLUNNS SAME LENGTH.

C SORT $X$ COLUMN AND KEEP ALL Y COLUMNS IN CORRESPONDENCE.

221 CALL LSURT ( $X X$, NX, YY, NY, NOYS, IFULL, NOYS)

0900000

0900010

0900020

0900030

0900040

0900050

0900060

0900070

0500080

0900090

0900100

0900110

0900120

0900130

0900140

0900150

0900160

0900170

0900180

0900190

0900200

0900210

0900220

0900230

0900240

0900250

0900260

0900270

0900280

0900290

0900300

0900310

0900320

0900330

0900340

0900350

0900360

0900370

0900380

0900390

0900400

0900410

0900420

0900430

0900440

0900450

0900460

0500470

0900480

0900490

0900500

0900510

0900520

0900530

0900540

0900550

0900560

0900570

0900580 





$C$ NOW COPY $X S$ INTO SECTION OF COMMON THAT CONTAINED Y $S$.

0901180

DO $255 \mathrm{~K}=1$, NYSUM

$K Z=N Y S U M+K$

$255 Z(K)=Z(K Z)$

C CALCULATE SCALING FACTOR FOR $X$.

$350 \quad S F X=($ XMAX $-X M I N) /$ FLOAT $($ NUML -1$)$

$S F X P R=I U . U * S F X$

C

C FIND MAX AND MIN FOR EACH Y COLUMN. $J=1$

$512 \operatorname{YMIN}(J)=0.0$

CALCULATE SCALING FACTORS FOR EACH Y COLUMN.

$S F Y(J)=4.0$

36. SFYPR $=10.0 * \operatorname{SFY}(J)$

C WRITE SCALES AND TITLES FOR EACH CURVE.

$500 \quad$ YSCALE $(1)=$ YMIN $(J)$

$L A M=2$

505 YSCALE $(L A M)=Y S C A L E(L A M-1)+S F Y P R$

$L A M=L A M+1$

IF $(L A M-12) 505,510,510$

$51 \mathrm{U}$ WRITE $(6,550) \mathrm{CHAR}(J), \operatorname{CHAR}(J), \operatorname{CHAR}(J),(Y T I T L E(K K, J), K K=1,8)$,

- (YSCALE(L), L = 1, 11 )

IF (J-NOYS $) \quad 511,600,600$

$511 \quad J=J+1$

GO TO 512

SET UP FOR LOOP TO PRINT ONE LINE OF GRAPH ON EACH PASS.

0901190

0901200

0901210

0901220

0901230

0901240

0901250

0901260

0901270

0901280

0901290

0901300

0901310

0901320

0901330

0901340

0901350

0901360

0901370

0901380

0901390

0901400

0901410

0901420

0901430

0901440

0901450

0901460

0901470

0901480

0901490

BOT $=$ TOP + SFX

C IFULL IS USED HERE TO STORE STOPPING POINT ON EACH Y COLUMN SCAN. DO 601 KK $=1$, NOYS

601 IFULL $(K K)=1$

C START LOOP FOR EACH LINE.

501500

0901510

0901520

0901530

DO 810 KLINE = I, NUML

C CLASSIFY KIND OF LINE.

C CLEAR LINE IMAGE TO BARE GRID OF USER S CHOICE.

IF( MOD ( KLINE, IO).EQ. I) GO TO 610

C THIS LINE CONTAINS NO SCALE INFORMATION.

IF ( KRID.EQ. 1 , GO TO 620

DO $630 \mathrm{KK}=1,91,10$

$\operatorname{LINE}(K K)=$ PLUS

DO $630 \mathrm{KJ}=1.9$

$K K J=K K+K J$

$630 \quad \operatorname{LINE}(K K J)=B L A N K$

0901540

0901550

0901560

0901570

0901580

0901590

0901600

0901610

0901620

0901630

0901640

GO TO 640

620 D0 621 KK $=1,99,2$

LINE $(K K)=$ PLUS

621 LINE $(K K+1)=$ BLANK

640 LINE(IUI) = PLUS

C RECORD TYPE OF LINE.

SLINE $=$.FALSE.

GO TO $650^{\circ}$

C THIS LINE CONTAINS SCALE INFORMATION.

610 DO $611 \mathrm{KK}=1,101$

611 LINE $(K K)=$ PLUS

C RECORD TYPE OF LINE.

0901650

0901660

0901670

0901680

0901690

0901700

0901710

0901720

0901730

0901740

0901750

0901760 
$\begin{array}{lr}\text { SLINE = .TRUE. } & 0901770\end{array}$




CIOO BLOCK DATA BLOCK DATA

C DEFINE TITLES AND PLOTTING SYMBOLS

DIMENSION YTITLE $(8,20)$, XTITLE $(30), \operatorname{CHAR}(5)$

COMMON/BPLOTI/YTITLE, XTITLE, CHAR

CHAR $(5)$

COMMON / BPLOT2/PLUS,BLANK

C

INTEGER PLUS, BLANK

CDATA (YTITLE $(K, 1), K=1,8) /$

$X \quad 48 H$ DISTANCE INCHES

2 (YTI TLE $(K, 2), K=1,8)$ )

$X \quad 48 \mathrm{H}$ DISTANCE INCHES

$4(Y T I T L E(K, 3), K=1,8) /$

$X \quad 48 \mathrm{H}$ DISTANCE INCHES

$X \quad(X T I T L E(K), K=1,30) /$

1000000

1000010

1000020

1000030

1000040

1000050

1000060

1000070

1000080

1,

1000090

$\times 66 \mathrm{H}$

1

1000100

,

1000110

1000120

$X \quad, 1 H B, I H U, 1 H R, 1 H N, 1 H O, 1 H U, 1 H T, I H, 1 H R, 1 H A, 1 H T, 1 H E, I H, 1 H(, 1 H /$, 1000130

1000140

1000150

$X 1 H K, 1 H O, I H D, I H /$,

XCHAR (1)/1H*/, CHAR(2)/1H(/,CHAR(3)/1H*/

C

DATA PLUS/1H+/,BLANK/IH,

END

1000160

1000170

1000180

1000190

1000200

$1000210-$ 


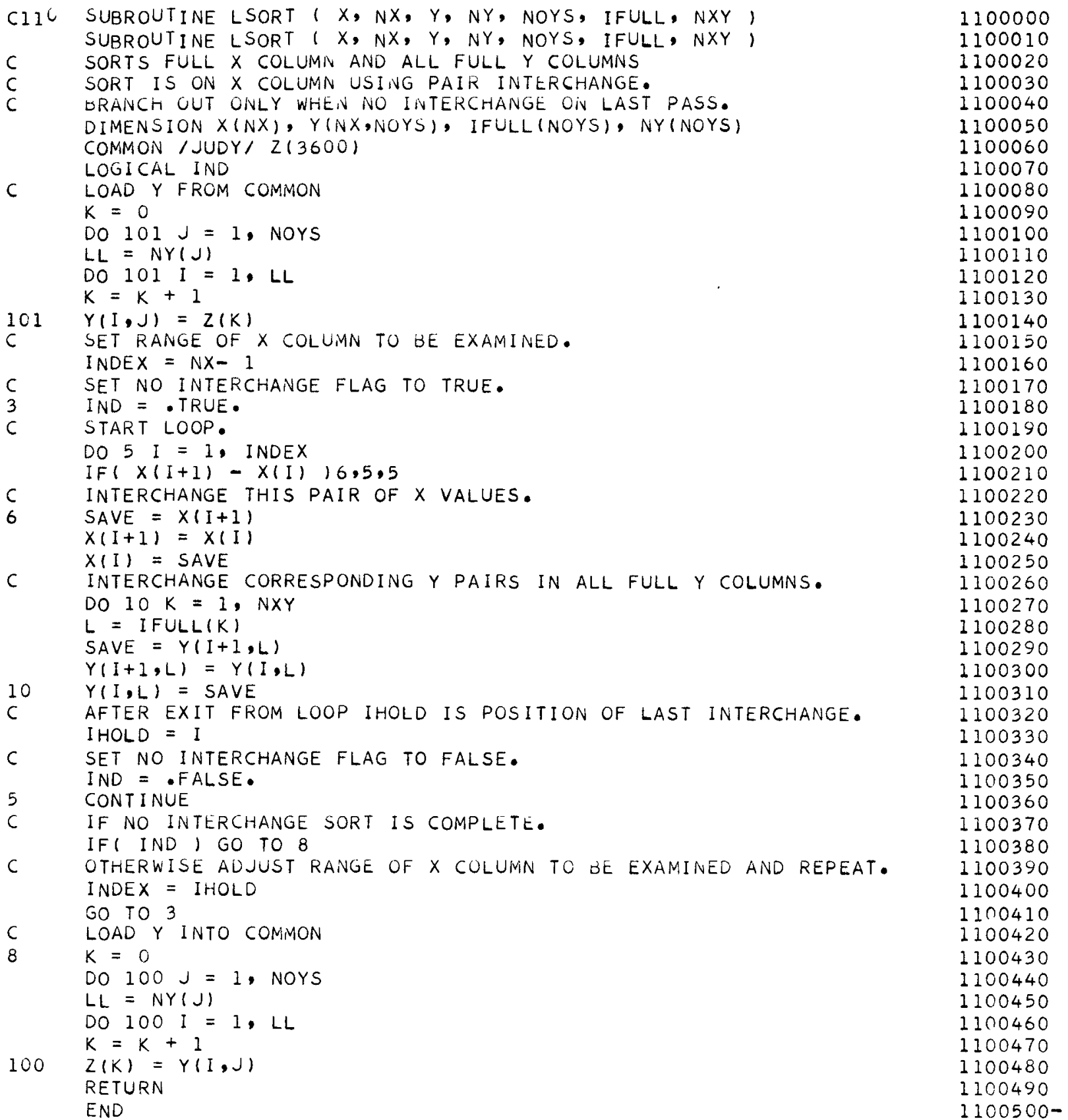




\begin{tabular}{|c|c|c|}
\hline $\mathrm{C} 120$ & $\begin{array}{l}\text { SUBROUTINE MSORT }(x, N X, Y, N Y, \text { NOYS, NI, TEMP }, \\
\text { SUBROUTINE MSORT }(x, N X, Y, N Y, \text { NOYS, NI, TEMP }\end{array}$ & $\begin{array}{l}1200000 \\
1200010\end{array}$ \\
\hline $\mathrm{C}$ & SORTS PARTIAL $X$ COLUMN IN TEMP AND ONE SHORT Y COLUMN. & $1<00020$ \\
\hline $\mathrm{C}$ & SORT IS ON TEMP USING PAIR INTERCHANGE. & 1200030 \\
\hline C & $\begin{array}{l}\text { BRANCH OUT WHEN NO INTERCHANGE ON LAST PASS. } \\
\text { DIMENSION } X(N X) \text {, Y(NX,NOYS), TEMP(NX), NY(NOYS) } \\
\text { CONMON /JUDY/ Z(3600) } \\
\text { LOGICAL IND }\end{array}$ & $\begin{array}{l}1200040 \\
1200050 \\
1200060 \\
1200070\end{array}$ \\
\hline$c$ & $\begin{array}{l}\text { LOAD Y FROM COMMON } \\
K=0 \\
D O 51 J=1, \text { NOYS } \\
L L=N Y(J)\end{array}$ & $\begin{array}{l}1200080 \\
1200090 \\
1200100 \\
1200110\end{array}$ \\
\hline & $\begin{array}{l}\text { DO } 51 I=1, \mathrm{LL} \\
K=K+1 \\
Y(I, J)=Z(K)\end{array}$ & $\begin{array}{l}1200120 \\
1200130\end{array}$ \\
\hline $\begin{array}{l}51 \\
C\end{array}$ & $\begin{array}{l}Y(I, J)=Z(K) \\
\text { LOAD FIRST NNY X VALUES INTO TEMP. } \\
\text { NNY = NY(NI) } \\
\text { DO } 100 I=1\end{array}$ & $\begin{array}{l}1200140 \\
1200150 \\
1200160 \\
1200170\end{array}$ \\
\hline $\begin{array}{l}100 \\
C \\
C\end{array}$ & $\begin{array}{l}\text { DO } 1001=1, \text { NNY } \\
\text { TEMP (I) }=X(I) \\
\text { SORT TEMP } \\
\text { SET RANGE OF TEMP TO BE EXAMINED. } \\
\text { INDEX }=\text { NNY }-1\end{array}$ & $\begin{array}{l}1200170 \\
1200180 \\
1200190 \\
1200200 \\
1200210\end{array}$ \\
\hline$C$ & SET NO INTERCHANGE FLAG TO TRUE. & 1200220 \\
\hline 3 & IND $=$-TRUE. & 1200230 \\
\hline C & $\begin{array}{l}\text { START LOOP. } \\
\text { DO } 5 \text { I }=1 ; \text { INDEX } \\
\text { IF }(\text { TEMP }(I+1)-\text { TEMP(I) } 16,5,5\end{array}$ & $\begin{array}{l}1200240 \\
1200250 \\
1200260\end{array}$ \\
\hline $\begin{array}{l}c \\
6\end{array}$ & $\begin{array}{l}\text { INTERCHANGE THIS PAIR OF TEMP VALUES. } \\
\text { SAVE = TEMP }(I+I)\end{array}$ & $\begin{array}{l}1200270 \\
1200280\end{array}$ \\
\hline & $\begin{array}{l}\text { TEMP }(I+I)=\text { TEMP (I) } \\
\text { TEMP }(I)=S A V E\end{array}$ & $\begin{array}{l}1200290 \\
1200300\end{array}$ \\
\hline C & $\begin{array}{l}\text { INTERCHANGE CORRESPONDING PAIR IN Y COLUMN NI. } \\
\text { SAVE = Y(I+I,NI) } \\
Y(I+I, N I)=Y(I, N I)\end{array}$ & $\begin{array}{l}1200310 \\
1200320 \\
1200330\end{array}$ \\
\hline C & $\begin{array}{l}\text { Y(I,NI) }=\text { SAVE } \\
\text { AFTER EXIT FROM LOOP IHOLD IS POSITION OF LAST INTERCHANGE. } \\
\text { IHOLD = I }\end{array}$ & $\begin{array}{l}1200340 \\
1200350 \\
1200360\end{array}$ \\
\hline C & $\begin{array}{l}\text { SET NO INTERCHANGE FLAG TO FALSE. } \\
\text { IND = FALSE. }\end{array}$ & 1200370 \\
\hline $\begin{array}{l}5 \\
c\end{array}$ & $\begin{array}{l}\text { CONTINUE } \\
\text { IF NO INTERCHANGE SORT IS COMPLETE. }\end{array}$ & $\begin{array}{l}1200390 \\
1200400\end{array}$ \\
\hline$C$ & $\begin{array}{l}\text { IF( IND I GO TO } 8 \\
\text { OTHERWISE ADUUST RANGE OF TEMP TO BE EXAMINED AND REPEAT. }\end{array}$ & $\begin{array}{l}1200410 \\
1200420\end{array}$ \\
\hline & $\begin{array}{l}\text { INDEX }=\text { IHOLD } \\
\text { GO TO } 3\end{array}$ & $\begin{array}{l}1200430 \\
1200440\end{array}$ \\
\hline C & LOAD Y INTO COMMON & 1200450 \\
\hline 8 & $\begin{array}{l}K=0 \\
D O 50 J=1, \text { NOYS } \\
L L=N Y(J) \\
D O 50 I=1, L L \\
K=K+1\end{array}$ & $\begin{array}{l}1200460 \\
1200470 \\
1200480 \\
1200490 \\
1200500\end{array}$ \\
\hline 50 & $\begin{array}{l}Z(K)=Y(I, J) \\
\text { RETURN } \\
\text { END }\end{array}$ & $\begin{array}{l}1200510 \\
1200520 \\
1200530\end{array}$ \\
\hline
\end{tabular}




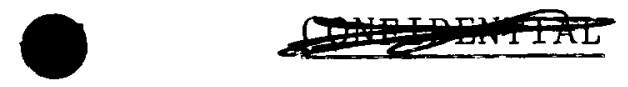

BNWL- 331

APPENDIX H

SAMPLE COMPUTER INPUT AND OUTPUT 
GOP IEST CASE - PARAMETER STUDY

\begin{tabular}{|c|c|c|c|c|c|}
\hline & TEST $-X$ & PLOTS & TRYOUT & $3-2-66$ & $3-2-66$ \\
\hline 10600 & 1991 & 1 & & & \\
\hline $\begin{array}{r}38 \\
83\end{array}$ & & 1788 & 1625 & 1125 & 875 \\
\hline 0 & & 5 & 28 & & \\
\hline 1 & 35 & & & & \\
\hline 3 & 55 & & & & \\
\hline $\begin{array}{l}1 \\
3\end{array}$ & $\begin{array}{r}79 \\
108\end{array}$ & & & & \\
\hline 2 & 118 & & & & \\
\hline 2 & 156 & & & & \\
\hline 3 & 185 & & & & \\
\hline 2 & 195 & & & & \\
\hline 3 & 215 & & & & \\
\hline$\frac{1}{3}$ & 266 & & & & \\
\hline 1 & 300 & & & & \\
\hline 3 & 320 & & & & \\
\hline 1 & 336 & & & & \\
\hline 166 & & 166 & 166 & & \\
\hline
\end{tabular}

426

$655000000+431900000+11000000000+0 \quad 000000000+0 \quad 350000000+1$ $-780000000+430,00000+12-307000000+496>000000+2000000000+0$ $-327000000+4608000000+4 \quad 793000000+4$

$-668000000+4295000000+4000000000+0$

$304000000+8 \quad 12400000-10 \quad 65100000-18 \quad 106000000-6 \quad 158000000+0$ $19220000+14 \quad 415000000-7 \quad 350000000-3 \quad 160000000-7 \quad 184000000+0$

$000000000+0000000000+0000000000+0000000000+0$

$000000000+0000000000+0000000000+0$

$000000000+n 000000000+0000000000+0 n 0000 n n 00+0$ nononnon +0

$000000000+0000000000+0000000000+0$

1



820 
GOP TEST CASE - PARAMETER STUDY

$\begin{array}{llll}\text { KEACIOR KEY } & \text { TEST }-X & \text { DATE CHARGED } & 3-2-66 \\ \text { CHANNEL NUMBER } & \text { PLOTS } & \text { DATE DISCHARGED } & 3-2-66\end{array}$

.0000 UPEN CHANNEL 35.0000 OPEN BOAT 55.0000 OPEN CHANNEL 79.0000 OPEN BOAT 108.0000 CLOSED BOAT 118.0000 UPEN BOAT 147.0000 CLUSED BOAT 156.0000 OPEN CHANNEL 168.0000

No. OH CHAMFER Flow Channels..............10600

NO. UH ANIVULAR FLUW CHANNELS............. 1995

Nu. Ot BURNOUT FLUW ChANNELS............ 9

REACTOK LATIICE SPACING............... 8.3580 ACIIVE LONE LENGTH (FLET)...............14.0000

EDGE OF CHAMFER Flow CHANNEL.......... 3890 DIAMETER OF EMPTY FLOW CHANNEL........ 1.7880 OUTSIDE DIAMETEK OF PROCESS TUBE....... 1.6250 OUTSIDE DIAMETER OF BOAT..............1.1250 INSIDE DIAMETER OF BOAT $. \cdots \cdots \cdots \cdots, .8750$ 
RATE CALCULATION PARAMETERS

\begin{tabular}{|c|c|c|c|c|}
\hline & \multicolumn{2}{|c|}{$\mathrm{CO}+\mathrm{H} 2 \mathrm{O}$} & \multicolumn{2}{|c|}{$\mathrm{Cn} 2+\mathrm{H}_{2}$} \\
\hline & 1 & 2 & 1 & \\
\hline $\begin{array}{l}\text { ARE UENCY FACTOR } \\
\text { INHIUITION FACTOK }\end{array}$ & $\begin{array}{l}-0.000 \\
-0.000\end{array}$ & $\begin{array}{l}-0.000 \\
-0.000 \\
-.0000\end{array}$ & $\begin{array}{l}-0.000 \\
-0.000\end{array}$ & $\begin{array}{l}-0.000 \\
-0.000 \\
-.0000\end{array}$ \\
\hline
\end{tabular}

CARBON DENSITIES

\section{MODERATOR $\quad 1.6600$}

BOAT $\quad 1.6600$

$\begin{array}{ll}\text { BOAT } & 1.6600 \\ \text { SAMPLE } & 1.6600\end{array}$

$$
\begin{aligned}
& \text { DIFFUSION } \\
& \text { FACTORS } \\
& \begin{array}{cr}
20 & 1.060-06 \\
\text { ATZ } 1.5800
\end{array} \\
& \text { OIFFUSION } \\
& \text { FACTORS }
\end{aligned}
$$

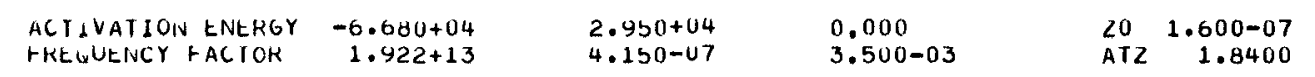

UATA FOK IHE LO + HZO REACTION IN A GAMMA FIELD
$G t=-0.000$
DELt $=-0.000$
XKRAD $=-0.000$
$A C O N=-0.000$

IPLOT $=1$ IEXIT $=0$ IETA= 1

UATA FUR THE COL + H2 REACTION IN A GAMNA FIELO

PRINT FREQ. $=1$

RGE $=-0.000 \quad$ RDELE $=-0.000 \quad$ RACON $=-0.000$

STEP SIZE $=6.0000+00$ UATA FOR OXYGEN - GRAPHITE REACTION
FREQUENCY FACTOR ACTIVATION ENERGY ORDER $\begin{array}{lccc} & \text { FREQUENCY FACTOR } & \text { ACTIVATION ENERGY } & \text { ORDER } \\ \text { THEKMAL } & \text { OLK10= }-0.000 & \text { O2E } 1=-0.000 & \text { APW }=-0.000 \\ \text { RAUIAIION } & 02 K 20=-0.000 & 02 E 2=-0.000 & \text { BPW }=-0.000\end{array}$ IEMPERATURES GAS 15.6 GRAPHITE INLET 500.00 MAXIMUM 1000.00

$2002=\begin{array}{lll}\text { DIFFUSION FACTORS } \\ -0.000 & \text { ABO2 }= & -0.000\end{array}$

EXTRAPOLATION DISTANCE 1.000

\footnotetext{
GAS 15.6 GRAPHITE INLET 500.00 MAXIMUM 1000.00
} 
GOP TEST CASE - PARAMETER STUDY

FLUH, PEKCENTAGES CHAMFER 85.482 ANNULAR 4.212 BURNOUT 10.306

IN1ILAL CONUITIONS FLOW $=285.00$ PHZO $=.500$ PCO $=20.750$ PCO2 $=361.760$ PH2 $=.076$ P02 $=.000$

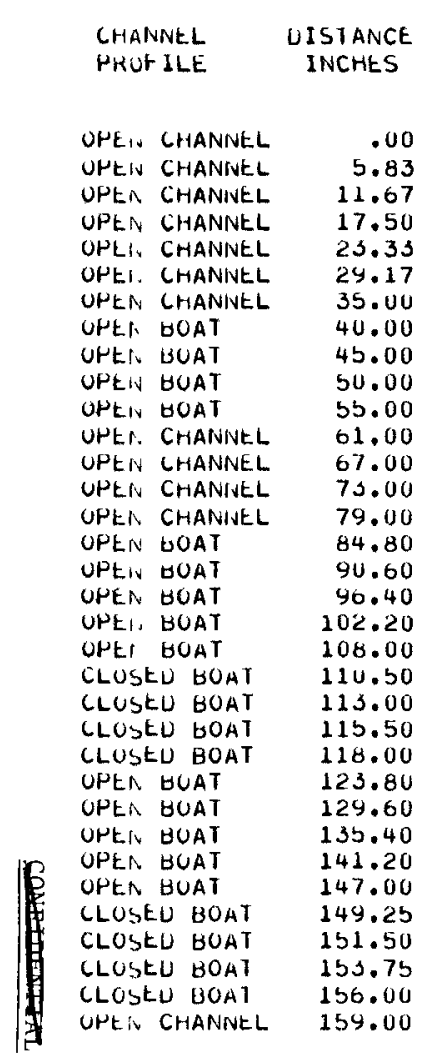

\begin{tabular}{|c|c|c|c|}
\hline $\begin{array}{l}P \text { A R R } \\
\mathrm{H}_{2} \mathrm{O}\end{array}$ & $\begin{array}{l}I \text { A L } \\
C U\end{array}$ & $\begin{array}{l}\mathrm{E} S \mathrm{~S} \\
\mathrm{H} 2\end{array}$ & $\mathrm{KEE} \underset{\mathrm{CO} 2}{\mathrm{~S}}$ \\
\hline 5000 & 20.7500 & .0760 & 361.7600 \\
\hline 5000 & 20.7500 & .0760 & 361.7600 \\
\hline .5000 & 20.7500 & .0760 & 361.7600 \\
\hline .5000 & 0.7500 & .0760 & 361.7600 \\
\hline .5000 & 0.7505 & .0760 & 361.7598 \\
\hline .4998 & 20.7544 & .0762 & 361.7579 \\
\hline .4992 & 0.7793 & .0768 & 361.7458 \\
\hline .4972 & 0.9660 & .0788 & 361.6534 \\
\hline .4934 & 21.4615 & .0826 & 361.4076 \\
\hline .4875 & 22.3625 & .0885 & 360.9600 \\
\hline .4792 & 23.6828 & .0968 & 360.3040 \\
\hline .4747 & 24.6824 & .1013 & 359.8064 \\
\hline & 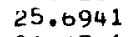 & .104 & .3023 \\
\hline .467 & 26.8 & .1087 & 358.7133 \\
\hline .4632 & 28.1800 & .1128 & 358.0634 \\
\hline .4457 & 31.4910 & .1303 & 356.4167 \\
\hline .4200 & 35.0684 & .1494 & 354.6375 \\
\hline .4094 & 38.3093 & .1666 & 353.0257 \\
\hline $.394 b$ & 41.0705 & .2815 & 351.6525 \\
\hline .3825 & $43 .<898$ & .1935 & 350.5489 \\
\hline .3797 & 43.9496 & .1903 & 350.2204 \\
\hline .3774 & 44.5 & .1986 & 349.9446 \\
\hline .3753 & 44.9 & .2007 & 349.7084 \\
\hline .37 & 45.37 & .2024 & 349.5128 \\
\hline .3695 & 46.0722 & .2005 & 349.1642 \\
\hline .3671 & 46.36 & & 349.0189 \\
\hline$x$ & 46.4417 & .2101 & 348.9813 \\
\hline .3653 & 46.4564 & .2107 & 348.9742 \\
\hline .3651 & 46.4599 & .2109 & 348.9725 \\
\hline .3651 & 46.4600 & .2109 & 348.9725 \\
\hline .3651 & 46.4600 & .2109 & 348.9725 \\
\hline .3651 & 46.4600 & .2109 & 348.9725 \\
\hline 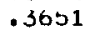 & 46.46 & .2109 & 348.9725 \\
\hline .3651 & 46.4600 & $.21 \cup 9$ & 348.9725 \\
\hline
\end{tabular}

02
.0000
.0000
.0000
.0000
.0000
.0000
.0000
.0000
.0000
.0000
.0000
.0000
.0000
.0000
.0000
.0000
.0000
.0000
.0000
.0000
.0000
.0000
.0000
.0000
.0000
.0000
.0000
.0000
.0000
.0000
.0000
.0000
.0000

TEMP. $-C$.

$B U R N O$

BOAT

SAMPLE

$.0000 \quad 500$

.0000 556. $-4.372-05 \quad 0.000 \quad 0.000$

$\begin{array}{llll}.0000 & -1.603-03 & 0.000 & 0.000\end{array}$

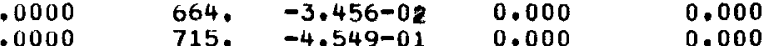

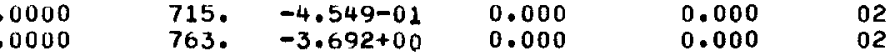



$\begin{array}{lllll}.0000 & 843 . & -6.318+01 & -9.411+01 & -9.388+01\end{array}$

876. $-1.351+02 \quad-2.680+02 \quad-2.667+02$

905. $-2.147+02-6.264+02-6.217+02$

930. $-2.880+02 \quad-1.242+03 \quad-1.230+03 \quad 04$

956. $-3.710+02 \quad 0.000 \quad 0.000$

976. $-4.468+02 \quad 0.000 \quad 0.000$

990. $-5.075+02 \quad 0.000 \quad 0.000$

998. $-5.439+0 z \quad 0.000 \quad 0.000$

1000. $\quad-5.435+02 \quad-6.465+03 \quad-6.370+03$

996. $\quad-5.136+02 \quad-5.642+03 \quad-5.563+03$

987. $-4.602+02 \quad-4.291+03 \quad-4.237+03$

952: $-3.140+02-1.688+03-1.573+03$

$9410-2.795+02 \quad-1.161+03 \quad-1.132+03$

930. $-2.438+02 \quad-8.342+02 \quad-8.148+02$

918. $-2.066+02 \quad-5.783+02-5.660+02$

905. $-1.680+02-3.859+02 \quad-3.788+02$

871. $-8.142+01-1.289+02 \quad-1.284+02$

832. $-2.542+01-3.278+01-3.274+01$

790. $-5.252+00 \quad-6.175+00 \quad-6.173+00$

744. $-7.791-01 \quad-8.330-01 \quad-8.329-01$

695. $-7.689-02$

675. -2.764-02

655. $-9.274-03$

635. $-2.900-03$

$\begin{array}{ll}614 . & -8.430-04 \\ 586 . & -1.439-04\end{array}$

$\begin{array}{ll}-7.766-02 & -7.766-02 \\ -2.775-02 & -2.775-02\end{array}$

$-9.287-03 \quad-9.287-03$

$-2.902-03 \quad-2.902-03$

$-8.431-04 \quad-8.431-04 \quad 02$

0.000 
GOP TEST CASE - PARAMETER STUDY

INIIIAL CUNUITIONS HLOW $=285.00$ PHZO $=.500$ PCO $=20.750$ PCO2 $=361.760$ PH2 $=.076$ PO2 $=.000$




DEPTH OF OXIDATION VALUES

ETA VALUES

\begin{tabular}{|c|c|c|c|c|}
\hline \multirow{2}{*}{\multicolumn{2}{|c|}{$\begin{array}{l}\text { CHAI.INEL } \\
\text { HROH ILE }\end{array}$}} & \multirow{2}{*}{$\begin{array}{l}\text { DISTAINCE } \\
\text { INCHES }\end{array}$} & \multicolumn{2}{|c|}{ WATER } \\
\hline & & & CHAN. & SAMP. \\
\hline UHEN & CHANINEL & b.8333 & 1.0000 & .0000 \\
\hline UPEN & CHANNNEL & 11.6067 & .9991 & .0000 \\
\hline urtivs & CHANNEL & 17.5000 & $.98<6$ & .0000 \\
\hline UPtiv & CHANNEL & 23.3333 & .8181 & .0000 \\
\hline UPES, & CMANIVELL & 29,1667 & .3069 & .0000 \\
\hline UPtin & CHANNEL & 35.0000 & $.1<13$ & .0000 \\
\hline UPE & BUAT & 40.0000 & .0585 & .9817 \\
\hline UPtiv & BUAT & 45.0000 & .0348 & .9570 \\
\hline UHEN & BOAT & 50.0000 & .0242 & .9223 \\
\hline UPE in 4 & BOAT & 55.0000 & .0188 & .8836 \\
\hline UPEN & CHANNEL & 01.0000 & .0151 & .0000 \\
\hline UPe & CHANINEL & 07.0000 & .0130 & .0000 \\
\hline UPE in & CHANIVEL & 73.0000 & .0119 & .0000 \\
\hline UPEIV & CHANIVEL & 79.6000 & .0113 & .0000 \\
\hline UPtiv & BUAT & 84.8000 & .0112 & .7560 \\
\hline OPtiv & BUAT & 90.6000 & .0115 & .7645 \\
\hline UPk +5 & BUAT & 96.4000 & $.01<3$ & .7842 \\
\hline UPER & OUAT & 102.2000 & .0137 & $.814 \theta$ \\
\hline OPtiv $t$ & BUAT & 100.0000 & .0103 & $.85 b 3$ \\
\hline CLUS,EL & $U$ GOAI & 110.5000 & .0179 & .5882 \\
\hline CLOSEL & D BOAT & 113.0000 & .0201 & .6406 \\
\hline LLUSEL & $\triangle B O A I$ & 115.5000 & .0230 & .6970 \\
\hline CLUSEL & $\triangle B O A T$ & 116.0000 & .0270 & .7569 \\
\hline UPtW & OUAT & 123.8000 & .0445 & .9713 \\
\hline UPEN & BUAT & $1<9.0000$ & .0925 & .9912 \\
\hline OPEIY & BUAT & 135.4000 & .2536 & .9982 \\
\hline UPE iv & BUAT & 141,2000 & .0676 & .9997 \\
\hline UPtew & BUAT & 147.0000 & .9511 & 1.0000 \\
\hline CLUSEL & U bOAT & 149.2500 & .9813 & 1.0000 \\
\hline LLO,EL & $\triangle B O A T$ & 151.5000 & .9935 & 1.0000 \\
\hline CLUSEL & $\triangle$ BOAT & 153.7500 & .9979 & 1.0000 \\
\hline CLUSEL & U BOAT & 156.0000 & .9994 & 1.0000 \\
\hline UPEIN & CHANIUEL & 159.0000 & .9999 & .0000 \\
\hline OPt I. & CHANIVEL & $16<.0000$ & 1.0000 & .0000 \\
\hline UPtiv & CHANIVEL & 105.0000 & 1.0000 & .0000 \\
\hline upt & CHANINEL & 168.0000 & 1.0000 & $.00 \cup 0$ \\
\hline & & & & \\
\hline & & & & \\
\hline & & & & \\
\hline
\end{tabular}

$\begin{array}{ll}.0000 & .0000 \\ .0000 & .0000 \\ .0000 & .0000 \\ .0000 & .0000 \\ .0000 & .0000 \\ .0000 & .0000 \\ .0000 & .0000 \\ .0000 & .0000 \\ .0000 & .0000 \\ .0000 & .0000 \\ .0000 & .0000 \\ .0000 & .0000 \\ .0000 & .0000 \\ .0000 & .0000 \\ .0000 & .0000 \\ .0000 & .0000 \\ .0000 & .0000 \\ .0000 & .0000 \\ .0000 & .0000 \\ .0000 & .0000 \\ .0000 & .0000 \\ .0000 & .0000 \\ .0000 & .0000 \\ .0000 & .0000 \\ .0000 & .0000 \\ .0000 & .0000 \\ .0000 & .0000 \\ .0000 & .0000 \\ .0000 & .0000 \\ .0000 & .0000 \\ .0000 & .0000 \\ .0000 & .0000 \\ .0000 & .0000 \\ .0000 & .0000 \\ .0000 & .0000 \\ .0000 & .0000\end{array}$

L(DEPTH) VALUES

$\begin{array}{lcc}\text { WATER } & \begin{array}{c}\text { CARBON } \\ \text { OIOXIDE }\end{array} & \text { OXYGEN } \\ 2.075+03 & 2.847+04 & 0.000 \\ 3.620+02 & 4.780+03 & 0.000 \\ 8.136+01 & 1.046+03 & 0.000 \\ 2.278+01 & 2.884+02 & 0.000 \\ 7.790+00 & 9.782+01 & 0.000 \\ 3.240+00 & 3.991+01 & 0.000 \\ 1.798+00 & 2.113+01 & 0.000 \\ 1.154+00 & 1.249+01 & 0.000 \\ 8.370-01 & 8.119+00 & 0.000 \\ 6.641-01 & 5.733+00 & 0.000 \\ 5.438-01 & 4.107+00 & 0.000 \\ 4.746-01 & 3.215+00 & 0.000 \\ 4.348-01 & 2.729+00 & 0.000 \\ 4.146-01 & 2.499+00 & 0.000 \\ 4.112-01 & 2.490+00 & 0.000 \\ 4.219-01 & 2.059+00 & 0.000 \\ 4.488-01 & 3.041+00 & 0.000 \\ 4.978-01 & 3.743+00 & 0.000 \\ 5.824-01 & 4.991+00 & 0.000 \\ 6.361-01 & 5.799+00 & 0.000 \\ 7.058-01 & 0.855+00 & 0.000 \\ 7.978-01 & 8.255+00 & 0.000 \\ 9.215-01 & 1.013+01 & 0.000 \\ 1.427+00 & 1.770+01 & 0.000 \\ 2.615+00 & 3.503+01 & 0.000 \\ 5.741+00 & 8.000+01 & 0.000 \\ 1.509+01 & 2.149+02 & 0.000 \\ 4.766+01 & 6.929+02 & 0.000 \\ 7.846+01 & 1.152+03 & 0.000 \\ 1.333+02 & 1.979+03 & 0.000 \\ 2.341+02 & 3.519+03 & 0.000 \\ 4.258+02 & 6.488+03 & 0.000 \\ 1.002+03 & 1.558+04 & 0.000 \\ 2.533+03 & 4.027+04 & 0.000 \\ 6.920+03 & 1.128+05 & 0.000 \\ 2.058+04 & 3.444+05 & 0.000 \\ & & \end{array}$

TEMP

OXYGEN
CHAN. SAMP.

CHAN. SAMP.

$1.0000 \quad .0000$

$1.0000 \quad .0000$

.9999 .0000

.9986 .0000

.9879 .0000

.7950 .9999

5825.9996

.3845 .9991

.1648 .0000

.1201 .0000

.0976 .0000

$.0874 \quad .0000$

$.0870 \quad .9903$

$.0945 \quad .9915$

.1461 .9935

$.2121 \quad .9976$

.2569 .9922

$.3158 \quad .9944$

$.3916 \quad .9962$

$.4845 \quad .9974$

.91341 .0000

.98201 .0000

99981.0000

99991.0000

1.00001 .0000

1.00001 .0000

1.00001 .0000

1.00000

$1.0000 \quad .0000$

$1.0000 \quad .0000$

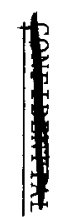

556.

611.

664.

715.

763.

808.

843.

876.

930.

956.

976.

990.

998.

1000 .

996.

972.

952.

941.

930.

918.

905.

871.

832.

790.

$744^{\circ}$

695.

675.

635 .

614.

586.

558 .

529. 


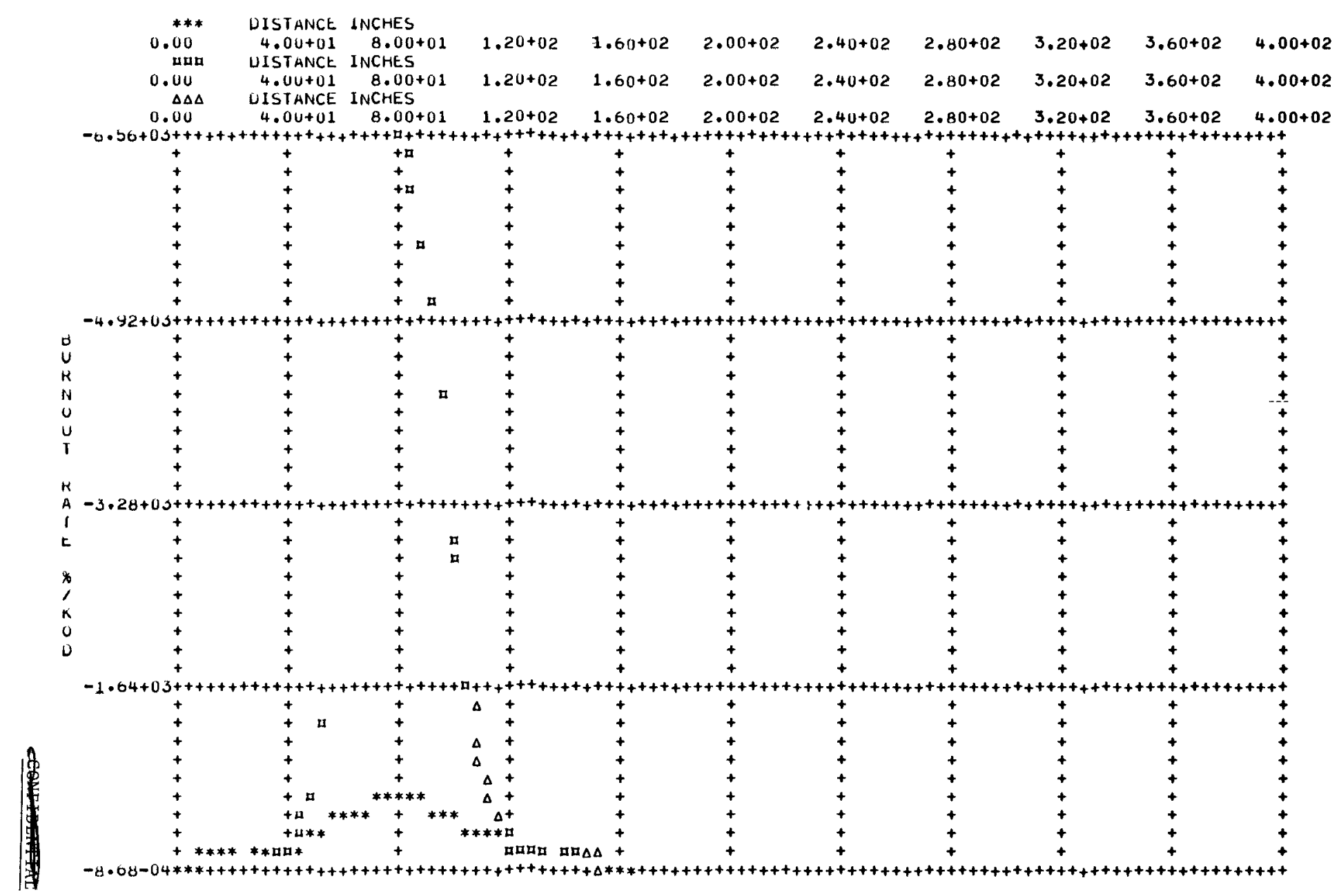




\section{REFERENCES}

1. R. C. Giberson, and J.P. Walker. "Reaction of Nuclear Graphite with Water Vapor--Part I: Effect of Hydrogen and Water Vapor Partial Pressures," Carbon, vol. 3, pp. 521-525 (1966).

2. P. L. Walker, Jr., et al. "Gas Reactions of Carbon," Advances in Catalysis, II, pp. 133-221 (1959).

3. W. M. Graven and F. J. Long. "Kinetics and Mechanisms of the Two Opposing Reactions of the Equilibrium $C O$ $+\mathrm{H}_{2} \mathrm{O}=\mathrm{CO}_{2}+\mathrm{H}_{2}$,"J. Am. Chem. Soc., vol 76, p. 2602, p. 6421 (1954).

4. G. L. Tingey. "Kinetics of Water-Gas Equilibrium Reaction. I. The Reaction of Carbon Dioxide with Hydrogen," J. Phys. Chem. vol. 70, pp. 1406-1412 (1966).

5. G. L. Tingey. "Radiolysis of Mixtures of Carbon Monoxide with Water Vapor," HWSA-3440, Apriz 16, 1964.

6. G. L. Tingey. The Radiolysis of a Mixture of Carbon Dioxide and Hydrogen, BNWL-SA-591. April 1966.

7. Peter Henrici. Discrete Variable Methods in Ordinary Differential Equations, John Wiley and Sons, Inc., pp.120-123 (1962).

8. J. Ralston and H. S. Wilf (editors), Mathematical Methods for Digital Computers, John Wiley and Sons, Inc., pp.99-100 (1960). 


\section{DISTR IBUTION}

Number of Copies

ACF Industries, Inc.

AEC Patent office

Aerojet-General Corporation (NASA)

Aerojet-General Corporation, Sacramento

Aerojet-General Nucleonics

Aerojet-General Nucleonics (NASA)

Aeronautical Systems Division

Air Force Electronic Systems Division

Air Force Technical Applications Center

Albuquerque Operations Office

Al1ison Division-GMC

Argonne National Laboratory

Army Ballistic Research Laboratories

Army Chief of Research and Development

Army Materials Research Agency

Army Nuclear Defense Laboratory

Army Picatinny Arsenal

Army Reactors Field Group

Army Weapons Command

Atomic Energy Commission, Washington

Atomic Energy Commission, Washington

Division of Reactor Development and Technology

J. E. Fox

R. E. Pahler

J.M. Simmons

Atomic Energy Commission, Washington

Division of Production

W. J. Lindsey

F. P. Self

C. W. Showalter

Atomic Energy Commission, Richland

Office of Senior RDT Site Representative

P. G. Holsted (2)

L. R. Lucas

Atomic Power Development Associates, Inc.

Atomics International

Battelle Memorial Institute

Brookhaven National Laboratory

Bureau of Mines, Albany

Canoga Park Area Office

Carolinas-Virginia Nuclear Power Associates, Inc. 
Number of

Copies

2

1

1

2

1

1

3

1

3

1

1

1

1

1

8

1

2

3

1

1

1

3

1

1

1

1

1

1

3

1
Central Intellıgence Agency

Chicago Patent $r$ nup

Combustion Engineering Inc.

Combustion Engineering. Inc (NRD)

Division of Internation Affairs, Brussels

Douglas AIrcraft Company. Inc; Newport Beach

Douglas United Nuclear

$$
\begin{array}{ll}
\mathrm{P} & \mathrm{A} \text { Carison } \\
\mathrm{M} & \mathrm{T} \text { Lewis } \\
\mathrm{A} & \text { Russeli }
\end{array}
$$

Dow Chemical Company, Rocky Flats

du Pont Company, Aiken

du Pont Company. W: Imington

EG\&G. Inc Dubiin

EGEG. Inc Goleta

EG\&G; Inc, Las Vegas

General Atomic Division

General Atomic Division

$$
\begin{array}{lll}
\text { W } & \text { E } & \text { Beil } \\
\text { R } & \text { D } & \text { Burnette } \\
\text { D } & \text { R } & \text { Lofing } \\
\text { C } & \text { A } & \text { Perrey } \\
\text { V } & \text { H } & \text { Pierce } \\
\text { R } & \text { F } & \text { Turner } \\
\text { E } & \text { O } & \text { Winkier }
\end{array}
$$

General Dynamics/Electric Boat Division

General Dynamics/Fort Worth

General Electric Company: Cincinnatı

General Electric Company (FPD)

General Electric Company (MSVD)

General Electrıc Company; Richland

$$
\text { D } \mathrm{H} \text { Curtiss }
$$

General Electric Company, San Jose

Goodyear Atomic Corporation

IIT Research Institute

Institute for Defense Analyses

Iowa State University

Jet Propulsion Laboratory

Johns Hopkins University (APL)

Knol1s Atomic Power Laboratory

Lockheed Georgia Company

Los Alamos Scientific Laboratory 
Number of Copies
Los Alamos Scientific Laboratory

P. G. Salgado

Martin Company

Materia1s Advisory Board

Metals and Controls, Inc.

Mound Laboratory

MSA Research Corporation (COO-765)

NASA Langley Research Center

NASA Lewis Research Center

NASA Manned Spacecraft Center

NASA Marshal1 Space Flight Center

National Aeronautics and Space Administration, Washington

National Lead Company of Ohio

National Reactor Testing Station (INC)

Naval Ordnance Laboratory

Naval Radiological Defense Laboratory

Naval Research Laboratory

Naval Ship Systems Command Headquarters

Naval Weapons Laboratory

Navy Marine Engineering Laboratory

New Brunswick Area Office

New York Operations Office

Nuclear Materials and Equipment Corporation

Oak Ridge Operations Office

Office of the Chief of Naval Operations

Pratt and Whitney Aircraft Division (NASA)

Public Health Service

Radio Corporation of America, Cranbury

Rand Corporation

Reactive Metals, Inc., Ashtabula

Republic Aviation Division

Richland Operations Office

W. Devine/R. M. Midkiff

M. H. Arndt/D. W. Mazur

O. H. Rathbun/W. C. Gallaugher

Sandia Corporation

Sandia Corporation, Livermore

Tracerlab, Richmond

TRW Systems

Union Carbide Corporation (ORGDP)

Union Carbide Corporation (ORNL) 
Number of Copies

1

1
Union Carbide Corporation (ORNL)

W. B。Cottrel1

T。S。Kress

R。E. MacPherson

F。H。Neill

W。C 。 U1rich

Union Carbide Corporation (Paducah Plant)

Union Carbide Corporation ( $\mathrm{Y}-12$ Plant)

University of California, Berkeley University of California, Livermore Westinghouse Bettis Atomic Power Laboratory Westinghouse Electric Corporation Westinghouse Electric Corporation, Lima Westinghouse Electric Corporation, Lima (AF) Westinghouse Electric Corporation (NASA) White Sands Missile Range Division of Technical Information Extension

Batte11e-Northwest

$R$. Y。 Dean

D。R。deHalas

R。C.Giberson (1)

R。 E。 Nightingale

C.A. Oster

G。L T Tingey

Technical Information Files (3)

Technical Publications 